\title{
3D reconstruction of the spatial distribution of dislocation loops using an
}

\section{automated stereo-imaging approach}

\author{
Hongbing $\mathrm{Yu}^{1}$, Xiaoou $\mathrm{Yi}^{2}$, Felix Hofmann ${ }^{1 *}$ \\ (1) Department of Engineering Science, University of Oxford, Parks Road, Oxford, OX1 3PJ, \\ UK \\ (2) School of Materials Science and Engineering, University of Science and Technology Beijing, \\ Beijing, 100083, China
}

\begin{abstract}
We propose an automated stereoscopic imaging approach for reconstructing the 3D spatial distribution of small dislocation loops (DLs) from 2D TEM micrographs. This method is demonstrated for small DLs in tungsten, formed by low-dose ion-implantation, that appear as circular spots in diffraction contrast TEM images. To extract the 3D position of specific DLs, their 2D position in multiple weak-beam dark-field TEM micrographs, recorded at different tilt angles, is fitted. From this fit the geometric centre and size of each DL in each micrograph can be extracted. To identify each specific DL in all the 2D projections, an automated forward prediction approach is used. A system of linear equations can then be setup, linking the 3D position of each DL to its $2 \mathrm{D}$ position in each projection, and solved using least-squares fitting. This approach is initially tested on synthetic data. For low projected loop densities $\left(<20 \times 10^{15} \mathrm{~m}^{-2}\right)$ only 3 projections are required for perfect recovery of the defect microstructure. More projections are required when the projected number density increases or realistic errors are included. 3D reconstruction of experimental data from the lowdose self-ion implanted tungsten sample reveals a damage microstructure in good agreement with the depth-dependent damage profile predicted by SRIM. A comparison with weighed back-projection shows that the stereo-imaging-approach requires fewer projections, is less sensitive to the angular
\end{abstract}

\footnotetext{
* Corresponding author: felix.hofmann@eng.ox.ac.uk
} 
range spanned, and is more resilient to spurious variations in local contrast. It also allows a more straightforward retrieval of quantitative information such as size and position of each loop.

Keywords: Dislocation loops, TEM, 3D spatial distribution, stereo-imaging.

\section{Introduction}

Irradiation-induced nano-scale lattice defects play a key role in the degradation of mechanical and thermal transport properties of materials used in nuclear reactors ${ }^{1,2}$. The evolution of the size and density of these defects at low irradiation doses (before the overlap of displacement cascades) provides an opportunity to gain insight into the fundamental mechanisms governing the formation and evolution of irradiation-induced damage ${ }^{3-5}$. Transmission electron microscopy (TEM) is one of the most powerful tools to observe the formation and evolution of these few nanometer large defects ${ }^{6,7}$. For instance, in-situ TEM irradiation, has been used extensively to reveal the dynamics of lattice defects such as dislocation loops (DLs) and stacking fault tetrahedra (SFT) formed during the collapse of displacement cascades in the early stage of irradiation ${ }^{3,5,8,9}$. Weak-beam dark-field (WBDF) TEM techniques have been widely used to characterize the size, Burgers vector and nature of DLs and SFT at different dose levels ${ }^{3,5,7,10-12}$. They are generally considered to provide the most accurate size information for small irradiation-induced lattice defects ${ }^{13}$. However, TEM micrographs are only 2D projections of the $3 \mathrm{D}$ volume. The position information in the thickness direction is lost, severely hampering the analysis of $3 \mathrm{D}$ defect interactions.

Experimental observations ${ }^{14}$ and defect simulation ${ }^{15,16}$ have shown that elastic interactions between lattice defects play an important role in the microstructural evolution of ion-irradiation induced damage in tungsten. These elastic interactions depend on the relative 3D positions of defects, as well as their distance from the sample surface. Thus far, defect positions in these studies have been estimated solely by considering the separation of defects in 2D TEM micrographs, neglecting the depth information ${ }^{14,16}$. Furthermore, ion irradiation produces a non-uniform damage distribution in the 
depth direction. This effect should be accounted for when considering the evolution of microstructure with ion fluence, however, due to a lack of 3D spatial resolution, this is not generally done ${ }^{3,5,9}$.

Stereo microscopy has been used to evaluate the depth of dislocations in TEM foils of neutronirradiated metals as early as the $1960 \mathrm{~s}{ }^{17-20}$. It involves the use of 2 images of the sample, recorded from different angular positions, to determine, by triangulation, the depth of specific defects. However, even today it is still not widely adopted. This is in large part because stereo microscopy requires the manual pairing of dislocation loops in TEM micrographs recorded from different tilting angles. The shift of the dislocation loop in two stereo images, relative to some surface decoration, is then determined using the parallax bar and the $3 \mathrm{D}$ position estimated. This is a laborious process, particularly if 2D coordinates of dislocation loops have to be read from hard copy TEM images. Use of digital TEM micrographs, recorded using a CCD camera, makes it somewhat easier to extract the 2D coordinates of paired dislocation loops. Very recently, a 3D reconstruction of spatial distribution of dislocation loops, without consideration of dislocation loop size, has been made for ion-implanted tungsten based on the stereo-imaging approach ${ }^{21}$. However, even here, manually paring of dislocations and acquisition of coordinates were required. This process is time intensive and subjected to human errors, making it unsuitable for repeatable analysis of large numbers of dislocations loops and samples. Thus, to facilitate gaining a more complete understanding of the formation and evolution of irradiation-induced damage, approaches that allow fast, accurate and consistent determination of the 3D spatial distribution of defects are urgently needed.

Numerous tomography techniques have been proposed to recover 3D microstructure from a series of 2D projection images. Back-projection and simultaneous iterative reconstruction techniques (SIRT) are perhaps the most widely used for bright field tomography, particularly on biological samples ${ }^{22,23}$. For high quality reconstructions they require a large number of projections (more than 100) collected over a wide range of tilting angles (at least $\pm 60^{\circ}$ ) with $\sim 1^{\circ}$ or smaller angular step size ${ }^{24,25}$. In TEM tomography high tilting angles are problematic as they increase the electron path length through the sample and cause shadowing effects when conventional TEM holders and samples are used ${ }^{25}$. Indeed, even if large tilt angles are reached, both back-projection and SIRT still suffer from "missing 
wedge" artefacts. In the last two decades, advanced reconstruction schemes such as discrete algebraic reconstruction technique ${ }^{24,26}$ (DART) and total variation minimization ${ }^{26-29}$ (TVM) method have been introduced. These methods effectively address the missing wedge problem and yield acceptable reconstructions, even for a reduced number of projections, by making use of prior knowledge.

For the characterization of crystalline materials, and particularly lattice defects within them, the use of TEM techniques that rely on diffraction contrast is attractive. However, diffraction contrast from crystals generally does not satisfy the projection requirement and can cause substantial artefacts when conventional reconstruction approaches are used ${ }^{25}$. As a result, the application of 3D TEM tomography to imaging of crystal defects via their associated strain fields has only enjoyed limited success. For example, careful experiments, where the scattering vector (g) and deviation parameter $\left(\mathrm{sg}_{\mathrm{g}}\right)$ were held constant for a number of tilt angles, have been successfully used to image 3D structures of discrete dislocations from both WBDF or bright field (BF) images ${ }^{30-33}$. For very small defects, such as small DLs formed in low dose implanted metals, a further complication is that the contrast is very sensitive to subtle changes in $\mathrm{s}_{\mathrm{g}}$, as well as changes of the depth of the DL in the TEM foil ${ }^{4,34}$. In pure $\mathrm{W}$ it has been reported that even a slight change in $\mathrm{s}_{\mathrm{g}}$ or loop depth can result in the transition of a small loop from visible to invisible, as well as alter loop size and morphology ${ }^{5,10}$. This means that sample tilt alone, or any local deviation from the desired diffraction conditions due to e.g. localized bending, sample quality or small rotations, can change the morphology, size and visibility of a DL. This experimental uncertainty means that the image of the same DL, recorded at different tilt conditions, will most certainly violate the projection principle. Furthermore, changes in $\mathrm{s}_{\mathrm{g}}$ and loop movement under electron illumination ${ }^{3,5}$ can also cause imperfect alignment of projections, leading to blurring and an overestimation of defect size in $3 \mathrm{D}$ reconstructions.

Here we propose an automated stereo-imaging approach to address this challenge. First, using a forward prediction method, the image associated with each specific DL is identified in all WBDF micrographs of the same sample volume recorded at different tilts. A system of linear equations is then set up linking the $3 \mathrm{D}$ position of each DL to its $2 \mathrm{D}$ position in each projection. Since more than 2 projections are used, this system of equations is overdetermined and least-squares fitting is employed 
to find the 3D position of each DL. The robustness of this reconstruction approach is explored using synthetic test data. Considering self-ion damaged tungsten as an example, the results of the stereoimaging method are compared to the 3D damage microstructure recovered using weighted backprojection. This comparison reveals several advantages of the automated stereo-imaging method.

\section{Materials and Method}

\subsection{Materials}

TEM tomography data, i.e. 2D micrographs of DLs at different tilt angles, was recorded from a 150 keV self-ion implanted ultra-high purity W TEM foil (99.999\% at. wt.). The sample was thinned to electron transparency by twin-jet electropolishing, using an electrolyte of $0.5 \mathrm{wt} \% \mathrm{NaOH}$ aqueous solution close to $0{ }^{\circ} \mathrm{C}$, and a polishing voltage of $14 \mathrm{~V}$. This was followed by 3 rinses in methanol. Insitu irradiation was performed with $150 \mathrm{keV}$ W-ions at a temperature of $30 \mathrm{~K}$ to a fluence of $1.25 \times$ $10^{12} \mathrm{ion} / \mathrm{cm}^{2}$ on the IVEM-Tandem facility, Argonne National Laboratory, USA. The damage dose corresponds to $\sim 0.01$ displacements per atom (dpa) at the peak of the damage profile according to SRIM calculation (quick K-P mode, displacement energy $=68 \mathrm{eV}$ ) ${ }^{35}$. Data was collected in the asirradiated condition and following a warm-up of the sample to room temperature. Here we use the data collected at room temperature. Using a double tilt holder, TEM micrographs were recorded under $\mathrm{a}(\mathbf{g}, 5.25 \sim 6.25 \mathbf{g})$ weak-beam dark-field condition with $\mathbf{g}=(110)$. The grain under consideration had approximately [001] surface normal, and the specimen was tilted around both $\alpha$ and $\beta$ tilting axes from $\alpha=-36^{\circ}, \beta=20.2^{\circ}$ to $\alpha=32^{\circ}, \beta=-19.6^{\circ}$. This is equivalent to tilting around the $\mathbf{g}$ vector from $41^{\circ}$ to $37^{\circ}$. Care was taken to ensure that the $\mathbf{g}$ vector was maintained as (110) and the WBDF condition unchanged. This is important, as both the $\mathbf{g}$ vector and deviation parameter $\left(\mathrm{s}_{\mathrm{g}}\right)$ will affect the visibility and apparent size of DLs. Overall, 19 WBDF TEM micrographs were recorded at different tilting conditions, as shown in supplementary figure S1, with a constant pixel size of $0.43 \mathrm{~nm}$ by $0.43 \mathrm{~nm}$. It should be noted that $0.43 \mathrm{~nm}$ is the pixel size at the magnification at which images were recorded, not the resolution of the WBDF technique. The resolution of WBDF images is 
governed by defect strain fields and sample quality, rather than the point-resolution of the microscope. For high quality foils, WBDF measurements are expected to resolve dislocation loops down to a size of $\sim 1 \mathrm{~nm}^{6,34}$.

\subsection{D stereo-imaging approach}

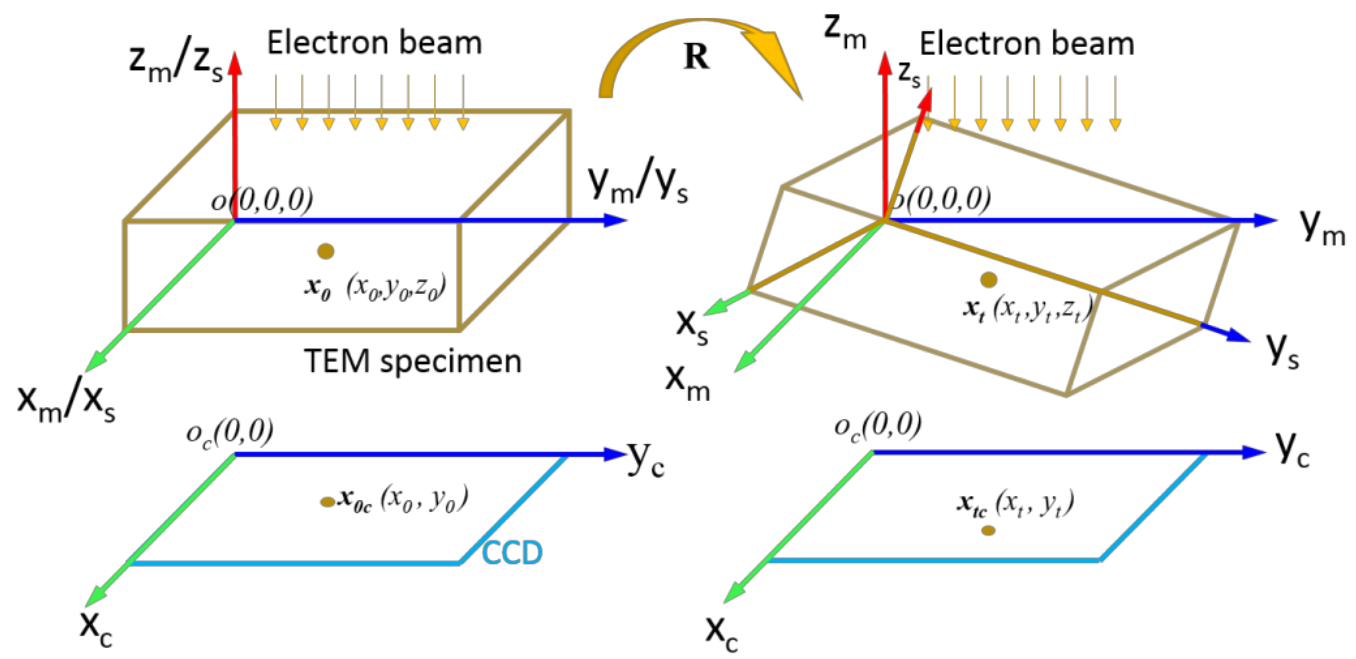

Fig. 1 Schematic diagram showing the geometric layout of electron beam, specimen, and CCD camera without and with tilting, and the definition of the coordinate systems of the specimen, microscope, and CCD camera.

To recover the 3D damage microstructure from the recorded 2D WBDF TEM micrographs, we propose the use of a stereo-imaging approach. Fig. 1 shows a diagram illustrating the coordinate systems attached to the microscope, camera, and specimen and how sample tilting modifies the position at which features in the sample will appear on the detector. A right-handed coordinate convention is used throughout. The $\mathrm{x}_{\mathrm{m}}-\mathrm{y}_{\mathrm{m}}-\mathrm{z}_{\mathrm{m}}$ the coordinate system is attached to the microscope, and its origin is placed at the rotation centre. The CCD camera has coordinate system $x_{c}-y_{c}$ that corresponds to a projection of the microscope's $\mathrm{x}_{\mathrm{m}}-\mathrm{y}_{\mathrm{m}}$ coordinates along the $\mathrm{z}_{\mathrm{m}}$ direction. That is, if a feature has a position $\mathbf{x}_{0}\left(\mathrm{x}_{0}, \mathrm{y}_{0}, \mathrm{z}_{0}\right)$ in microscope coordinates, its image on the CCD camera will be located at $\mathbf{x}_{0 c}\left(\mathrm{x}_{0}, \mathrm{y}_{0}\right)$. The specimen's coordinate system $\left(\mathrm{x}_{\mathrm{s}}-\mathrm{y}_{\mathrm{s}}-\mathrm{z}_{\mathrm{s}}\right)$ coincides with the microscope's system when no tilting is applied. In a TEM, the holder tilting axes generally do not 
coincide with the coordinate axes of the microscope or the CCD camera. Here we define the $\alpha$ (major) tilting axis as unit vector $\left[\mathrm{t}_{1}^{\alpha} \mathrm{t}_{2}^{\alpha} 0\right]$ and the $\beta$ tilting axis as unit vector $\left[\mathrm{t}_{1}^{\beta} \mathrm{t}_{2}^{\beta} 0\right]$, where both tilting axes are referenced to the microscope coordinate system for zero tilt. It should be noted that these two tilting vectors will vary from microscope to microscope. At the IVEM- Tandem facility, the $\alpha$ and $\beta$ tilting axes respectively are [- $\left.\begin{array}{lll}-0.90 & 0.44 & 0\end{array}\right]$ and $\left[\begin{array}{lll}0.44 & 0.90 & 0\end{array}\right]$. These tilts can be captured by a rotation matrix R:

$$
\mathbf{R}=\left[\begin{array}{lll}
R_{11} & R_{12} & R_{13} \\
R_{21} & R_{22} & R_{23} \\
R_{31} & R_{32} & R_{33}
\end{array}\right]=\mathbf{R}_{\alpha} \mathbf{R}_{\beta}
$$

where $\mathbf{R}_{\alpha}$ and $\mathbf{R}_{\beta}$ are the rotation matrices for the $\alpha$ tilt and $\beta$ tilt, respectively. The calculation of the matrix capturing rotation by a given angle about a unit vector is provided in Appendix 1.

After an arbitrary tilt, the coordinates, $\mathbf{x}_{\mathrm{t}}$, in the microscope frame of a DL at position $\mathbf{x}_{0}$ in the sample are given by:

$$
\mathbf{x}_{\mathrm{t}}=\mathbf{R} \mathbf{x}_{0}
$$

And coordinates of the DL in the CCD camera's coordinate system are

$$
\mathbf{x}_{\mathrm{tc}}=\widetilde{\mathbf{R}} \mathbf{x}_{0}
$$

where $\widetilde{\mathbf{R}}=\left[\begin{array}{lll}R_{11} & R_{12} & R_{13} \\ R_{21} & R_{22} & R_{23}\end{array}\right]$

If the coordinates of the DL, $\mathbf{x}_{0}$, are unknown, the position of the DL can be worked out from any two or more projections recorded at different, but known, tilting angles. If we have $\mathrm{N}(\mathrm{N} \geq 2)$ projections available, the coordinates of the DL in the $n$th $(\mathrm{n}=1,2,3, \ldots, \mathrm{N})$ projection are $\mathbf{x}_{\mathrm{tc}}{ }^{n}$, and the rotation matrix for the $n$th $(\mathrm{n}=1,2,3, \ldots, \mathrm{N})$ projection is $\mathbf{R}^{n}$, and a system of equations can be established which can be written in the form of the matrix,

$$
\mathbf{x}_{\mathrm{tc}}{ }^{N}=\mathbf{A} \mathbf{x}_{0},
$$

where 


$$
\mathbf{x}_{\mathrm{tc}}{ }^{N}=\left[\begin{array}{c}
{\left[\mathbf{x}_{\mathrm{tc}}{ }^{1}\right]} \\
{\left[\mathbf{x}_{\mathrm{tc}}{ }^{2}\right]} \\
\vdots \\
{\left[\mathbf{x}_{\mathrm{tc}}{ }^{N}\right]}
\end{array}\right] \text { and } \mathbf{A}=\left[\begin{array}{c}
{\left[\widetilde{\mathbf{R}}^{1}\right]} \\
{\left[\widetilde{\mathbf{R}}^{2}\right]} \\
\vdots \\
{\left[\widetilde{\mathbf{R}}^{N}\right]}
\end{array}\right]
$$

From this, it is clear that at least two projections are needed to recover $\mathbf{x}_{0}$. If $\mathrm{N}>2$ projections are acquired, the system of equations is over-determined. Here, least-squares fitting is used to find a solution for $\mathbf{x}_{0}$. This can be readily achieved using the pseudo-inverse of matrix $\mathbf{A}^{36}$.

$$
\mathbf{x}_{0}=\left[\left(\mathrm{A}^{\mathrm{t}} \mathrm{A}\right)^{-1} \mathrm{~A}^{\mathrm{t}}\right] \mathbf{x}_{\mathrm{tc}}{ }^{N}
$$

where $\mathbf{A}^{t}$ is the transpose of $\mathbf{A}$.

\subsection{Forward prediction}

It is straightforward to calculate the depth of a specific DL once the position of this DL in two or more projections is known. However, identifying the image associated with a specific DL in multiple different projections is challenging. Image cross-correlation ${ }^{37}$ and space invariant feature transformation ${ }^{38,39}$ are often used to match or recognize multiple instances of a specific feature ${ }^{40}$. However, there are many different DLs in each projection that have similar morphology, size, and intensity. Furthermore, the same DL will have slightly varying size, intensity, visibility and morphology when viewed in different projections as illustrated in supplementary figure S2. This complicates the use of image-correlation-based techniques for identifying the image of a specific DL in multiple projections. Instead, we propose a forward prediction approach to do the loop registration.

For any DL we can calculate the anticipated position in the camera coordinate frame if we know the 2D position of the DL in one projection and its depth in the sample coordinate frame. Since the depth of a given DL is not known a priori, we start with an initial guess of the depth. If the guess is close to the actual depth of the DL, the predicted position of the DL in other projections will match the position observed in the experimentally-recorded projections. However, if the guess is incorrect, the predicted 2D position for different projections will either not match the position of any DL or, wrongly, match the position of a different DL. Matching of the 2D position of a different DL will only occur sporadically. This means that the number of projections where a DL is found reaches a 
maximum when the guessed depth is close to actual depth value for that DL, as shown in Fig. 2. We note that a plateau rather than a single maximum point is found in the profile in Fig. 2. The best initial estimate of the loop depth is chosen as that with the smallest variance between predicted and measured 2D positions. Now each DL can be identified and labelled in all the projections. Then the system of linear equations described in Eq. (4) can be set up and solved using Eq. (5). Since the visibility of DLs may also change during tilting, even if $\mathbf{g}$ and the $\mathbf{s}_{\mathrm{g}}$ are kept constant, some DLs are only visible in a subset of projections. Here only loops that appear in 7 or more projections are considered.

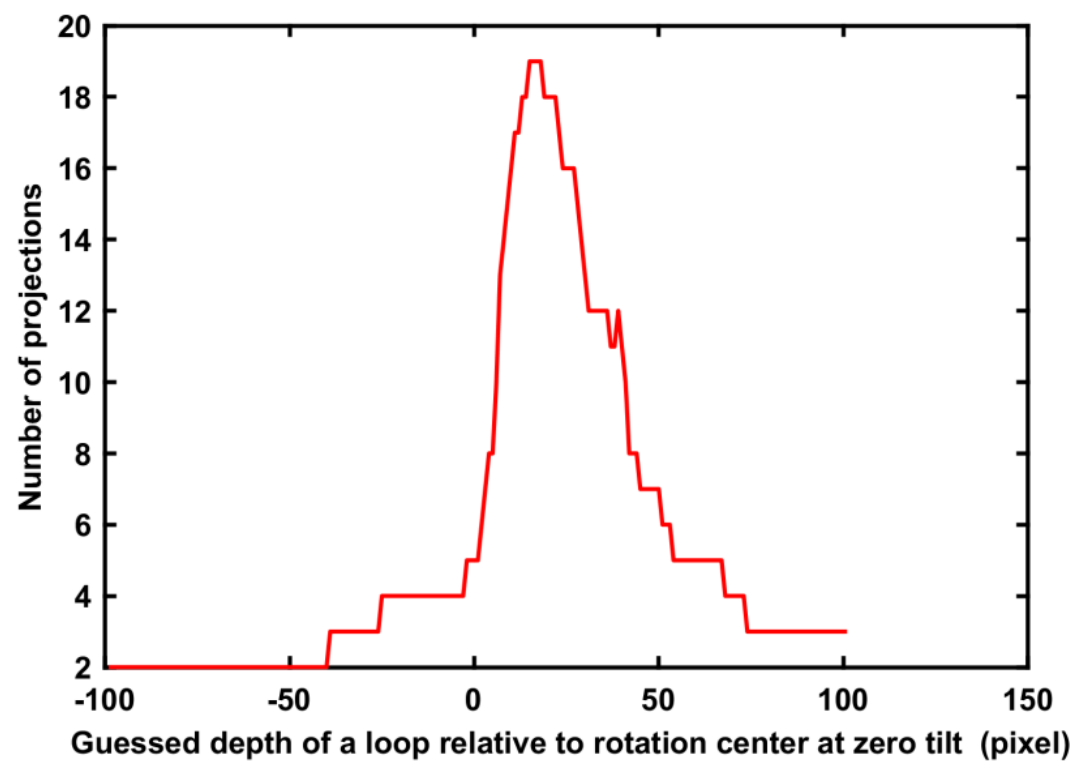

Fig. 2 The number of projections where a dislocation loop is matched versus the guess of depth of the dislocation relative to rotation center at zero tilt.

\subsection{Digital image processing}

Before 3D reconstruction, the raw WBDF TEM micrographs were pre-processed using the approach of Mason et al ${ }^{16}$ to remove the background signal. Variations in background intensity arise due to changes in foil thickness, foil surface topography and residual stress-induced sample bending. They make automated DL detection challenging as e.g. bright background signal may be counted as foreground, while a dim DL spot on a dark background may be ignored, if a global threshold is applied without first removing the background signal. The approach of Mason et al removes these 
background variations, while ensuring that the shape of foreground features is correctly retained (Fig 3 (b)). A detailed description of this algorithm is provided elsewhere ${ }^{16}$. Briefly, separation of the image into foreground and background is performed by considering the spatial wavelength of features. Bending and thickness imperfections are associated with long spatial wavelengths, while dislocation loops are associated with shorter wavelengths. For each pixel an estimate of the local average background intensity, and the probability that it should be treated as background is made. These estimates are iteratively refined until a stable estimate of the background signal is reached. Conceptually this approach is similar to blob-detection used in computer vision ${ }^{41}$. After removal of the background, a global threshold, determined using the Ridler \& Calvard ${ }^{42}$ thresholding algorithm, is applied to separate out the foreground features.

Next, each DL was fitted with a 2D Gaussian function, as shown in Fig. 3 (c), to determine the 2D position and size of each DL. From this a binary image of the DL population was produced, using the half maximum of the peak intensity of the fitted Gaussian function as the adaptive threshold for each fitted DL (Fig. 3(d)). 


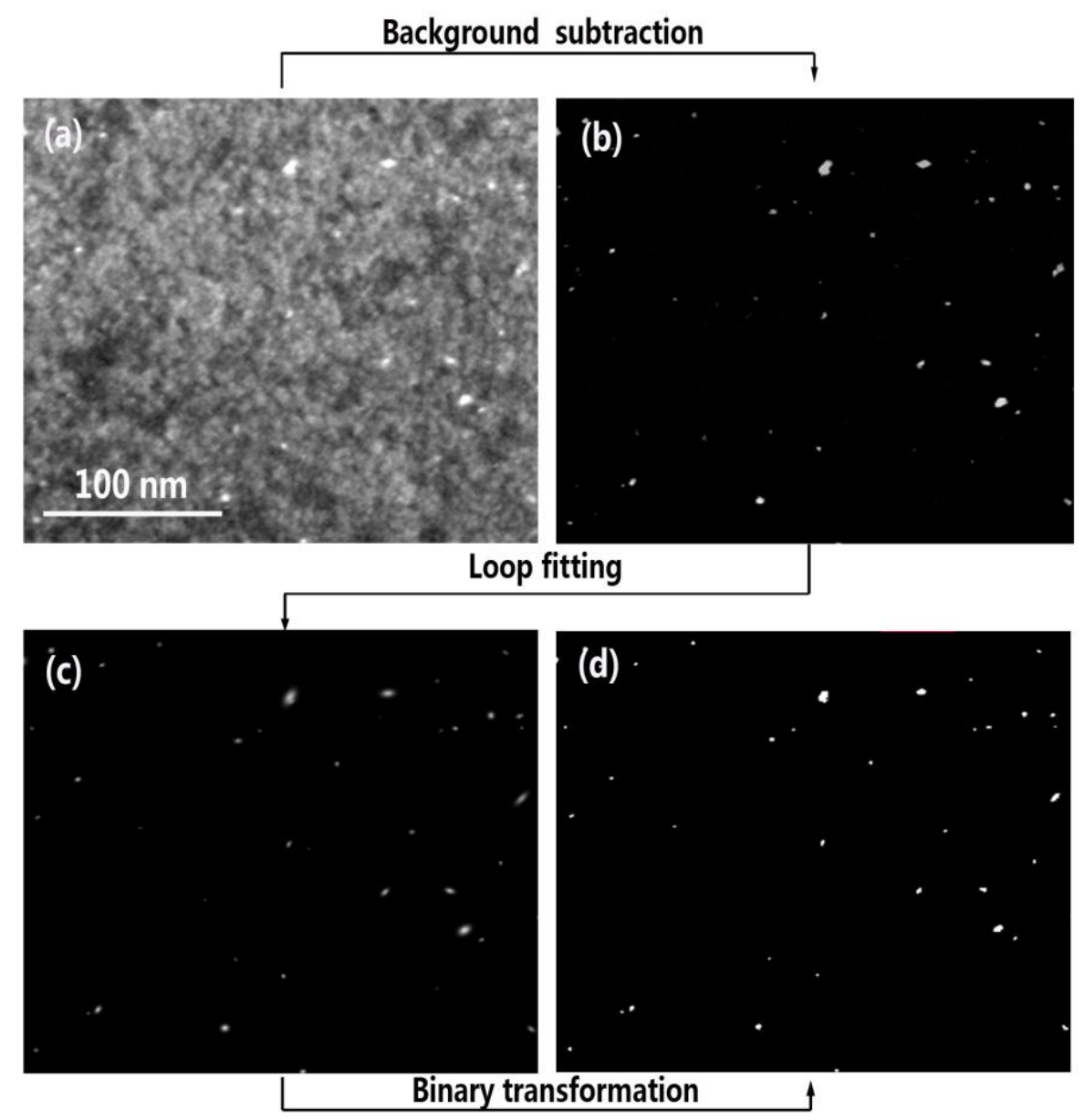

Fig. 3 Digital image processing. (a) Original image, (b) background subtracted, (c) dislocation loops with above-threshold intensity fitted with 2D Gaussian functions, (d) adaptive thresholding for each dislocation loop by half of the maximum intensity and conversion of the image to a binary image.

The last step before the forward prediction is to align all the micrographs to a common rotation centre. Here a DL prominently visible in all projections was chosen as the rotation centre. Using image crosscorrelation all micrographs were aligned such that the selected DL remained at the $(0,0)$ position. To reconstruct the 3D DL population, the forward prediction approach (section 2.3) was first used together with the binarized micrographs to perform the loop registration. Then, the accurate 2D position of each specific DL in each projection, determined using 2D Gaussian fitting, was used to determine the 3D position of each loop (section 2.2). 


\subsection{Back-projection}

To provide a comparison for the results of the stereo-imaging method, a 3D reconstruction using a generalized back-projection approach, was also considered. Back-projection refers to the process of projecting the intensity in a $2 \mathrm{D}$ projection back into the $3 \mathrm{D}$ volume along the projection path. By summing the back-projected intensity from multiple 2D projections, the 3D structure from which the 2D projections were acquired can be recovered. Fig. 4 (b) demonstrates, for a 2D example, the reconstruction by back-projection of the object in Fig. 4 (a), using a small number of projections (19) recorded at angles between $-40^{\circ}$ and $40^{\circ}$. Fig. 4 (b) clearly shows line-artefacts corresponding to the paths along which specific $2 \mathrm{D}$ projections were back-projected. These can be avoided by increasing the angular range covered by projections (ideally a full $180^{\circ}$ ) and reducing the angular interval between projections. A blurring of the reconstructed features is also evident, caused by a higher weighting of low-spatial frequencies during the back-projection process ${ }^{43}$.

This issue can be tackled by two different methods. The first is filtered back-projection which involves applying a frequency filter to the recorded projections to reduce low frequency components before back-projection ${ }^{43}$. The second is weighted back-projection where the point spread function associated with the back-projection is removed from the reconstructed $2 \mathrm{D}$ or $3 \mathrm{D}$ images using a deconvolution ${ }^{44}$. The point spread function can be obtained by forward-projecting a delta function (Fig. 5 (a)) and then back-projecting it in the same way as the object to be reconstructed. Fig. 5 (b) shows the 2D point spread function for the back-projection shown in Fig. 4 (b). A number of different algorithms can be used for the 3D deconvolution ${ }^{45}$. In this paper the Richard and Lucy iterative method was chosen because it provides a straightforward way of enforcing positivity of the deconvolution result. Fig.4 (c) and Fig. 4 (d) show reconstructions using filtered back-projection and weighted back-projection respectively of the image in Fig. 4 (a). Filtered back-projection substantially reduces the blurring of the reconstructed image, but the stripe artefacts, due to the small number of projections (19) and limited angular range, are still clearly visible. Weighted back-projection does a better job for a small number of projections. In this paper, weighted back-projection for dual-axis 
tilting was used to reconstruct the 3D spatial distribution of DLs for a comparison with the stereoimaging reconstruction approach.

Codes written for this paper are available for download DOI: 10.5281/zenodo.1402439.

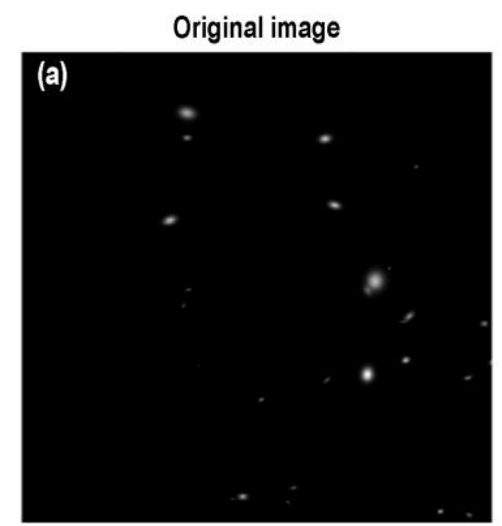

Filtered back-projection

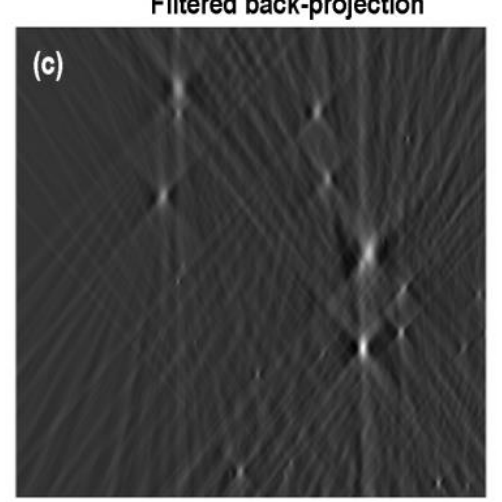

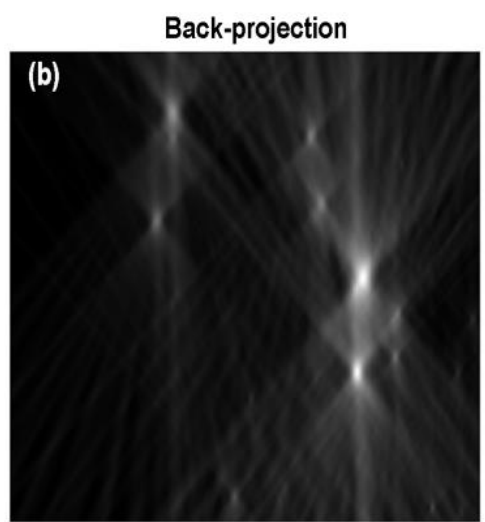

Weighted back-projection

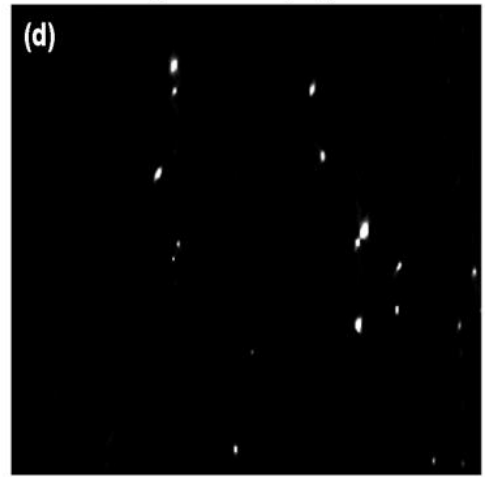

Fig. 4 A 2D demonstration of back-projection reconstruction and two methods to improve the quality of back-projection. (a) Original image, (b) back-projection from 19 projections recorded at angles ranging from $-40^{\circ}$ to $40^{\circ}$, (c) filtered back-projection, (d) deconvolution of the point spread function, shown in Fig. 5(b), from the back-projection reconstructed image. 

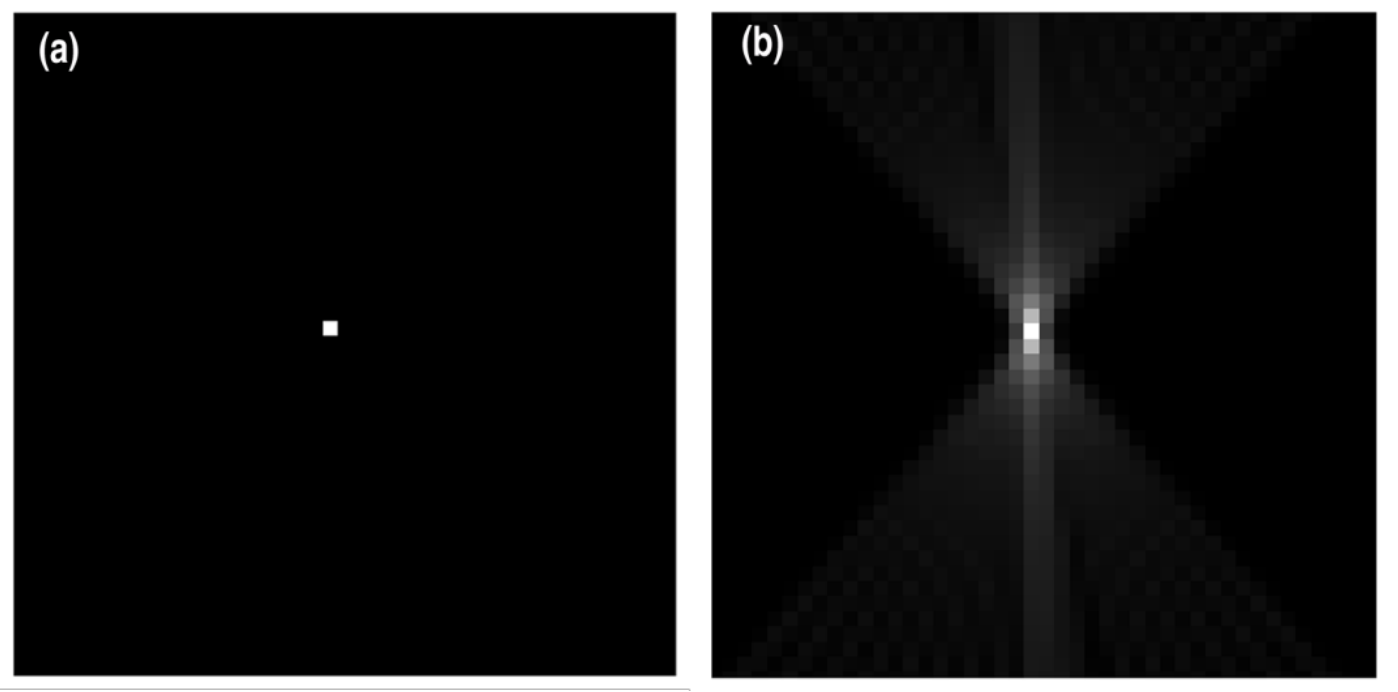

Fig. 5 (a) 2D delta function. (b) Reconstruction for the single point in (a) by back-projection from projections recorded at the same set of angles as in Fig. (4). 


\section{Results and discussions}

\subsection{Reconstruction of synthetic data}

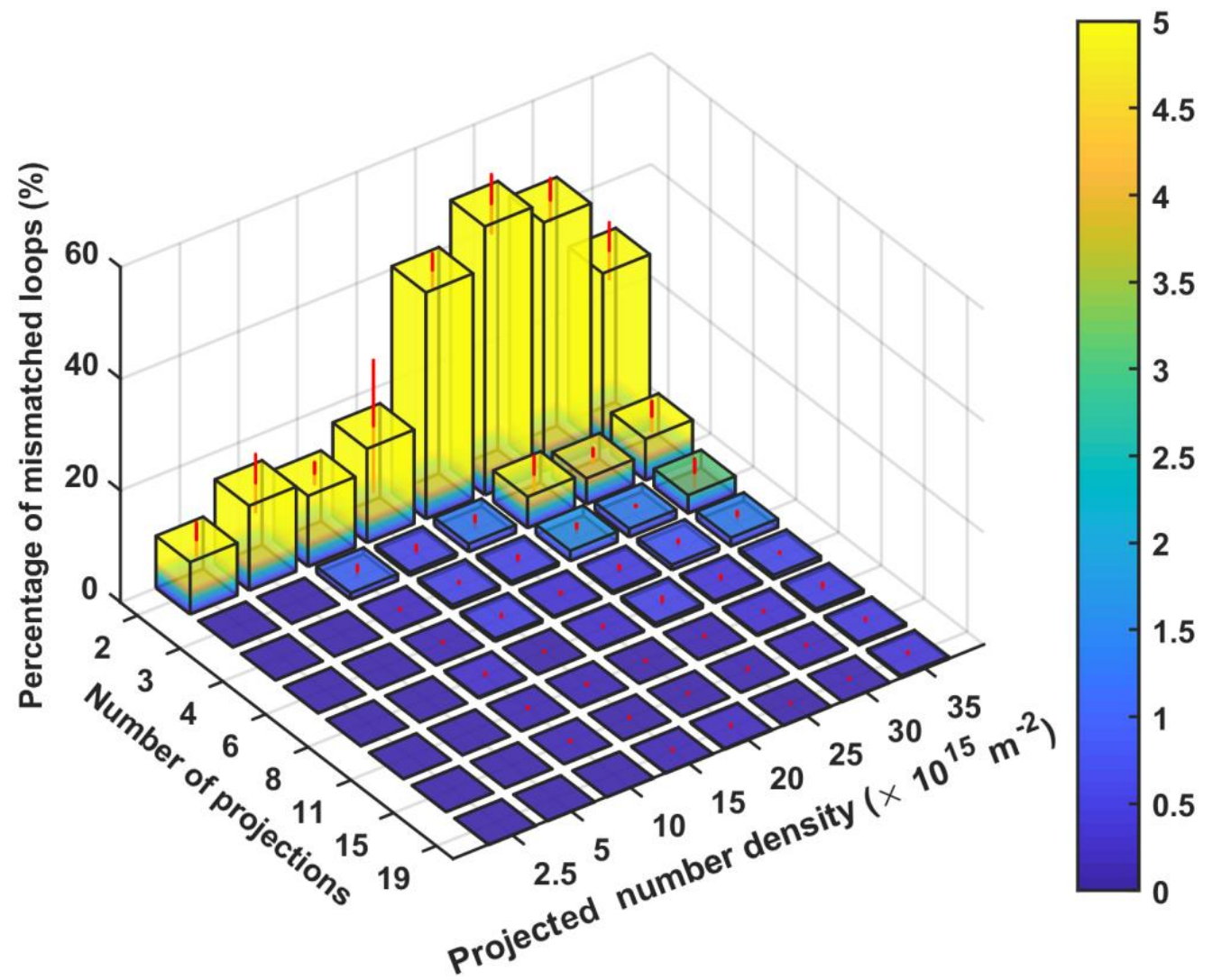

Fig. 6 The percentage of mismatched loops as a function of number of projections and projected number densities of loops for the case of perfect projections.

The automated stereo-imaging approach was initially tested on synthetic data. A 3D box of $200 \mathrm{~nm}$ by $200 \mathrm{~nm}$ by $100 \mathrm{~nm}$ with a variable number of DLs, ranging in size from $2 \mathrm{~nm}$ to $6 \mathrm{~nm}$, was created. The positions of the loops were randomly generated. The $3 \mathrm{D}$ volume was tilted to the same tilting angles used for acquisition of the experimental WBDF TEM micrographs. Then, the projection images were generated. These images were used to reconstruct the $3 \mathrm{D}$ defect population using the automated stereo-imaging approach. By varying the projected defect number density, and the number and combination of projections, the effect of these parameters on the quality of the reconstruction can be assessed. The projected densities were selected based on the saturation density of dislocation loops under heavy ion irradiation, which is about $5 \sim 12 \times 10^{15} \mathrm{~m}^{-2} 5,9,12$ dependent on irradiation temperature 
and impurities. To quantify reconstruction errors the recovered defects are compared with the ground truth, i.e. the defect population used to generate the synthetic projections. In particular, we compute the percentage of mismatched loops, average size error, and average position error. Mismatched loops have here been defined as loops where the distance between the reconstructed loop and the corresponding loop in the original volume is larger than $3 \mathrm{~nm}$. Each data point was obtained from 3 independent simulations.

Fig. 6 shows the percentage of mismatched loops as a function of the number of available projections $\left(\mathrm{N}_{\mathrm{v}}\right)$ and projected defect number density $\left(\rho_{\mathrm{p}}\right)$ for perfect projections. When only 2 projections are available, as is often the case in conventional stereo-imaging, a large proportion of loops are mismatched. The reason is that the constraint in the loop registration process, that a given DL must consistently appear in a number of reflections, is missing. Interestingly even just using 3 projections dramatically improves the situation. Here less than $1.5 \%$ loops are mismatched when $\rho_{\mathrm{p}}$ is lower than $20 \times 10^{15} \mathrm{~m}^{-2}$. When $\rho_{\mathrm{p}}$ increases further, reconstruction errors increase. Increasing $\mathrm{N}_{\mathrm{v}}$ substantially reduces these reconstruction errors and, for perfect projections, no more than 6 projections are needed. The average dislocation size error $(<0.003 \mathrm{~nm})$ and average position error $(<0.1 \mathrm{~nm})$ are small, as is evident from supplementary Fig. S3 and Fig. S4.

To simulate errors caused by imperfect projection, each individual loop is randomly shifted by $\Delta \mathrm{x}$ $(\Delta \mathrm{x} \in[-2,2] \mathrm{nm})$ and $\Delta \mathrm{y}(\Delta \mathrm{y} \in[-2,2] \mathrm{nm})$ along $\mathrm{x}$ and $\mathrm{y}$ direction in each projection as shown in the inset image of Fig. 7. These shifts are consistent with the maximum 2D position uncertainty estimated from the experimental data. Now the mis-matched loop error has increased significantly, as seen in Fig. 7, and more projections are required for a faithful reconstruction. When $\rho_{\mathrm{p}}$ is on the order of 2.5 $\times 10^{15} \mathrm{~m}^{-2}, 4$ or more projections are needed to achieve $<5 \%$ mis-matched loops. The minimum number of projections required for mismatch $<5 \%$ increases with increasing $\rho_{\mathrm{p}}$. For a density of 20 $\times 10^{15} \mathrm{~m}^{-2}$, which is much higher than the saturation density, 19 projections are required. The average position error and size error strongly depend on $\rho_{\mathrm{p}}$ as shown in supplementary Fig. S5 and Fig. S6. The average position error and size error are $\sim 0.7 \mathrm{~nm}$ and $\sim 0.027 \mathrm{~nm}$, respectively, when $\mathrm{N}_{\mathrm{v}}=19$ and $\rho_{\mathrm{p}}=20 \times 10^{15} \mathrm{~m}^{-2}$. 


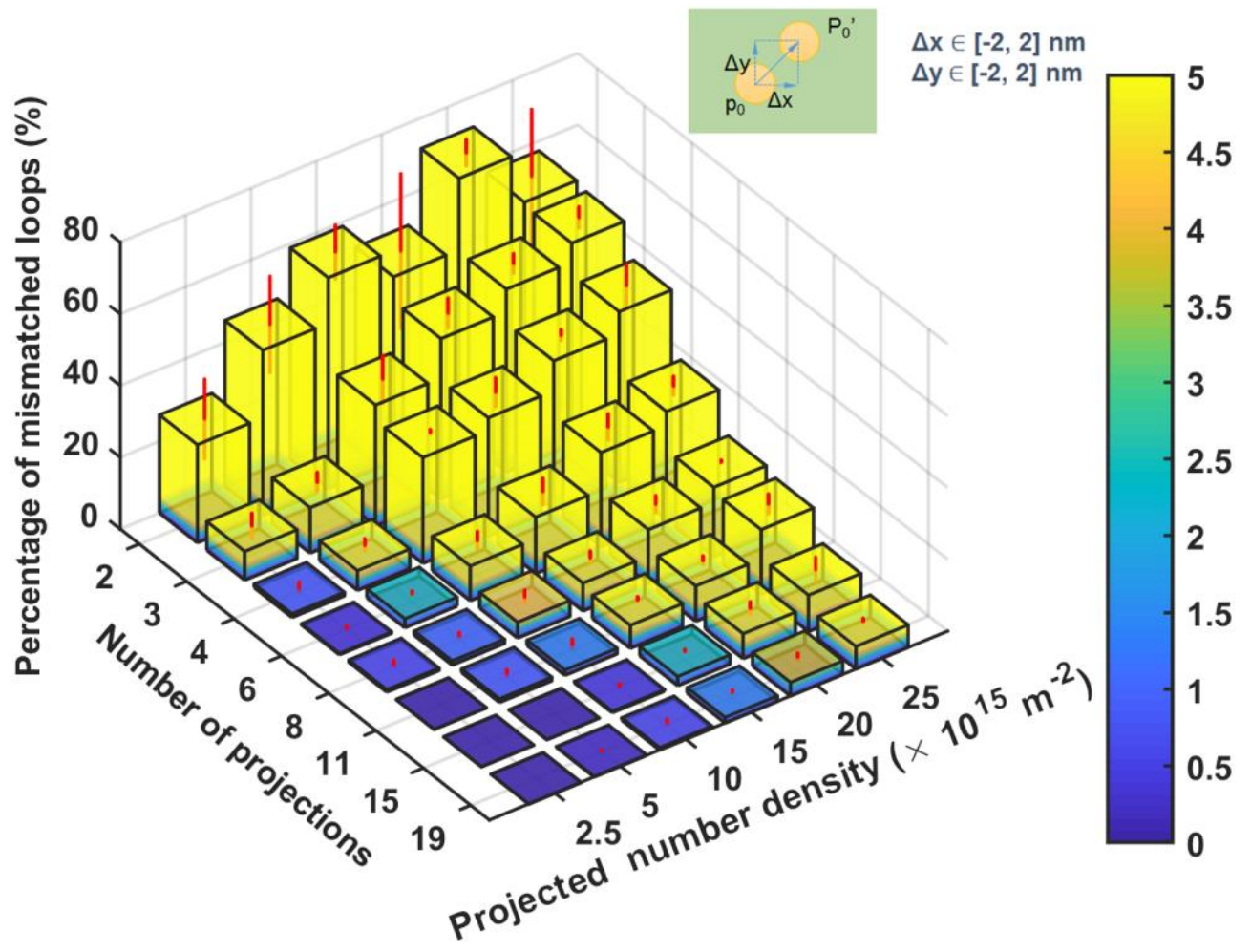

Fig. 7 The percentage of mismatched loops as a function of number of projections and projected number densities of loops for the case where random position shifts were added to the projections of loops.

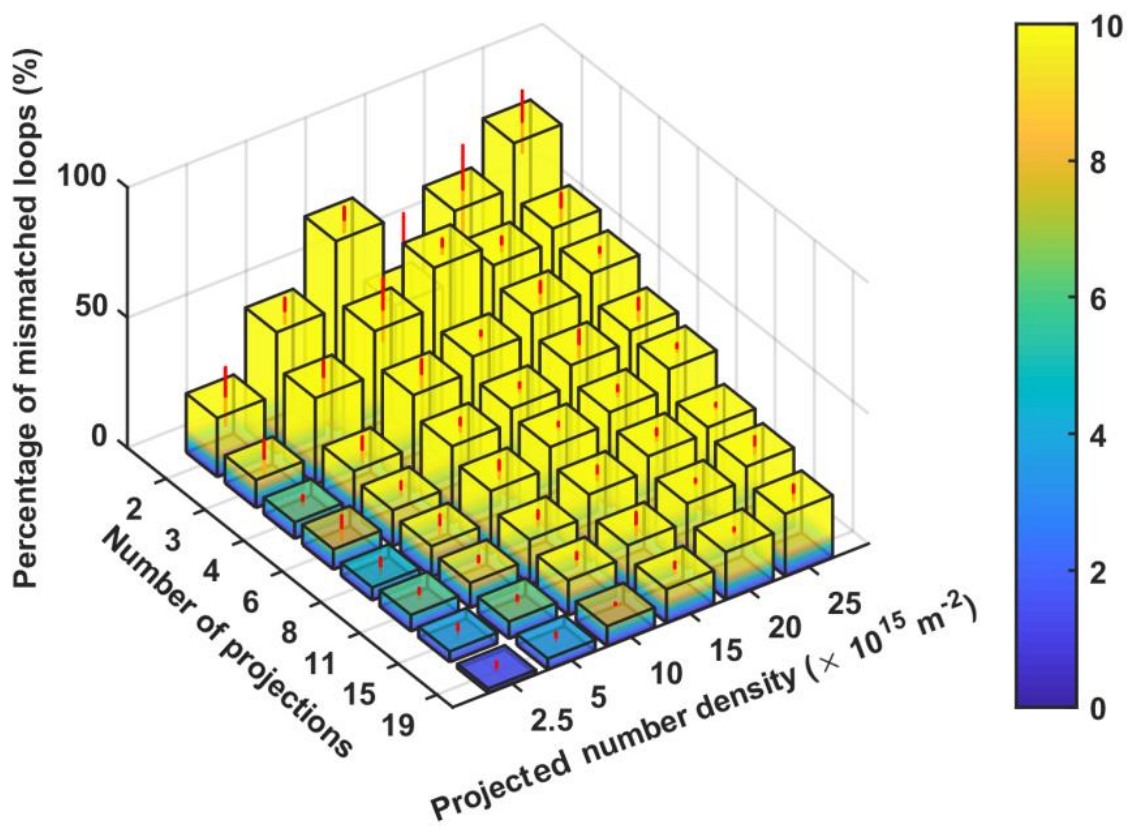

Fig. 8 The percentage of mismatched loops as a function of number of projections and projected number densities of loops for the case that random shifts plus variation in visibility across projections. 
As discussed above, the visibility of dislocation loops also changes across projections, as shown in supplementary Fig. S2. This change in visibility of dislocation loops will cause a further increase in reconstruction errors, as effectively data from fewer projections are available for the reconstruction of each loop. Here we make each dislocation loop disappear in randomly selected $\mathrm{n}(\mathrm{n} \in[0,11])$ projections. Fig. 8 shows the reconstruction errors when both random shift and variations in loop visibility are introduced into the synthetic data. In this case, at least $8 \sim 11$ projections are required for error $<5 \%$ when $\rho_{\mathrm{p}}$ is at the range of $2.5 \times 10^{15} \mathrm{~m}^{-2}$, and 19 projections when $\rho_{\mathrm{p}}$ at the range of $5 \times 10^{15}$ $\mathrm{m}^{-2}$. Fig. 9 shows the superposition of the synthetic data and the reconstructed 3D defect population from $\rho_{\mathrm{p}} \sim 5 \times 10^{15} \mathrm{~m}^{-2}$ and 19 projections (an animated version of this plot is provided in supplementary movie 1).

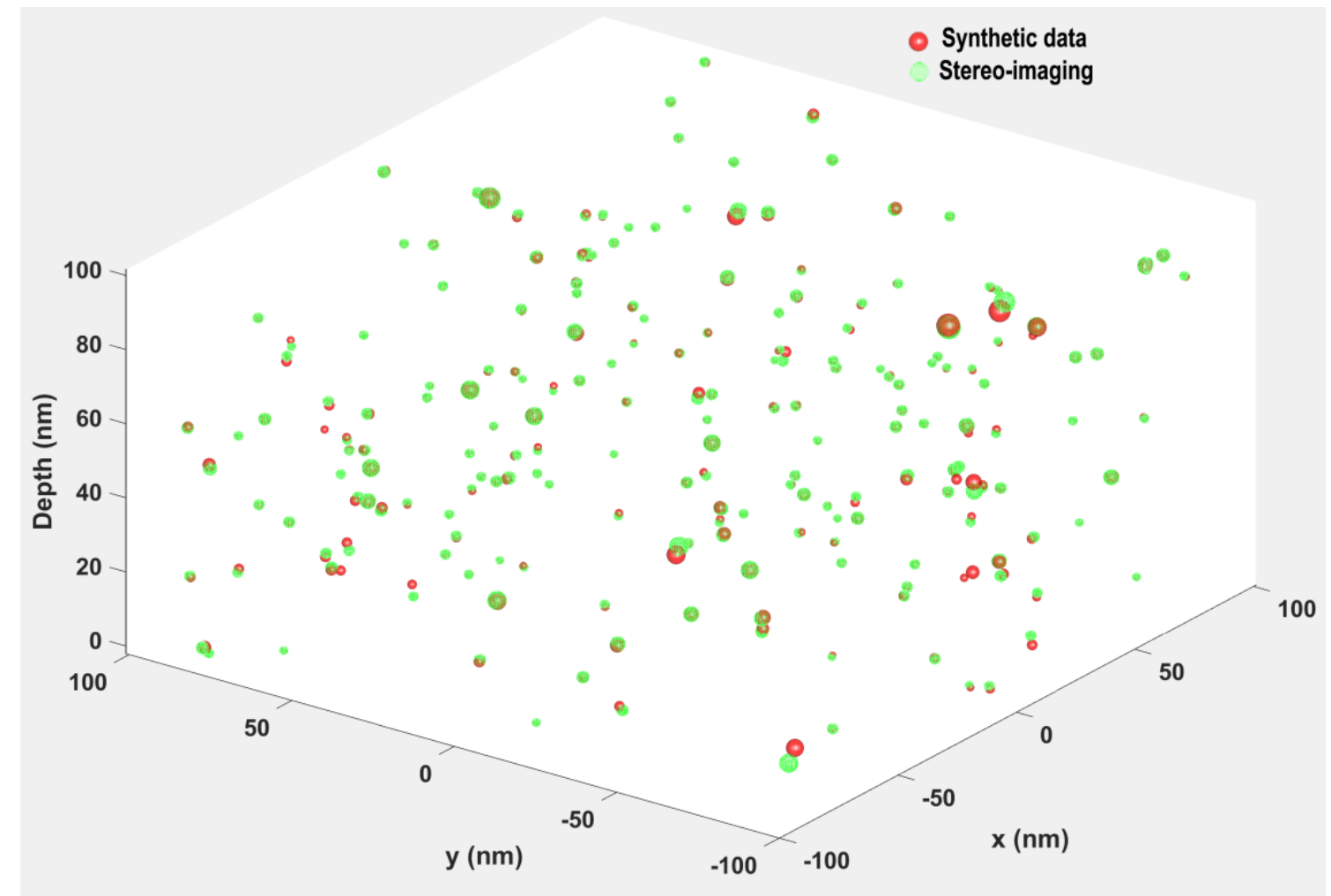

Fig. 9 Superposition of the synthetic data and the stereo-imaging reconstruction from 19 projections with random shift and variation in visibility $\left(\rho_{\mathrm{p}} \sim 5 \times 10^{15} \mathrm{~m}^{-2}\right)$. 


\subsection{Application to experimental data}

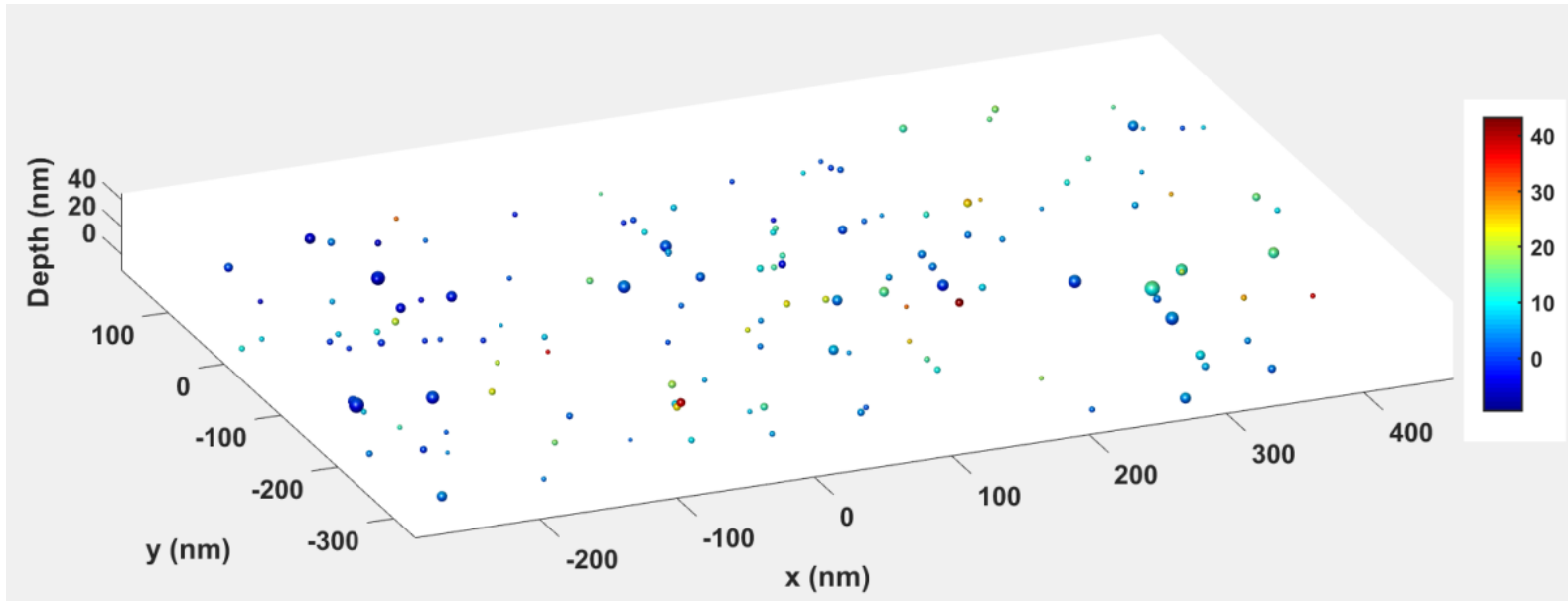

Fig. 10 3D spatial distribution of dislocation loops recovered from the experimental data using the automated stereo-imaging approach. The size is amplified by a factor of 2 for clearer visibility.

Fig. 10 shows the 3D spatial distribution of DLs recovered from the experimental series of 2D micrographs, recorded at different tilt angles, using the automated stereo-imaging approach. The projected number density of dislocation loops in the micrographs is $0.4 \sim 0.6 \times 10^{15} \mathrm{~m}^{-2}$. The spherical markers represent DLs in 3D space. For better visibility, their size has been amplified by a factor of 2 (an animated version of this plot is provided in supplementary movie 2). It can be clearly seen that DLs are confined to a shallow layer of $\sim 30 \mathrm{~nm}$ thickness, consistent with the damage layer thickness predicted by SRIM for irradiation of tungsten with $150 \mathrm{keV}$ self-ions (Fig. 12d). 
(a)

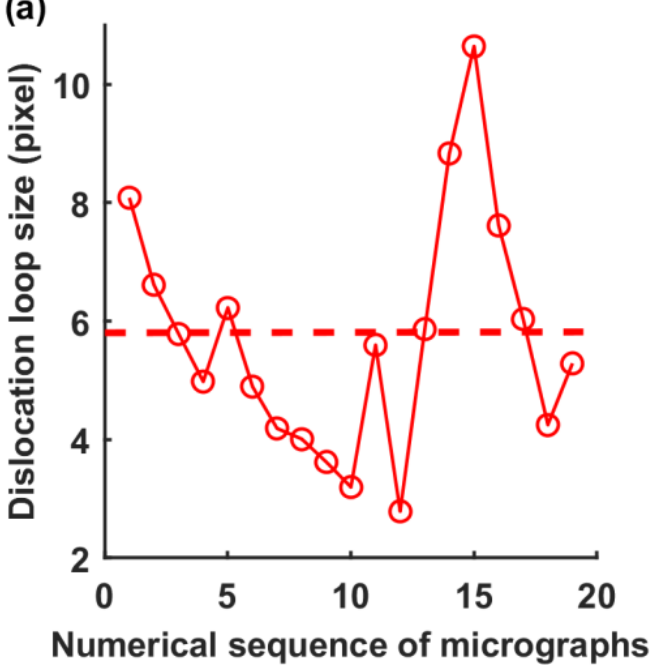

(b)

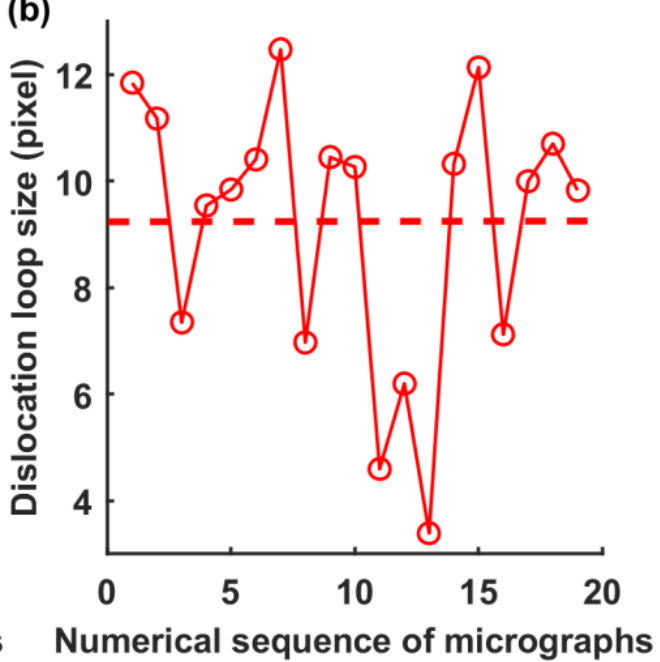

Fig. 11 Variation of the apparent size of two dislocation loops from projection to projection. The projections are ordered by increasing $\alpha$ tilting angle. Superimposed is a dashed line indicating the average size of each loop.

An important question concerns the determination of DL size. For small DLs, up to $10 \mathrm{~nm}$ in size, there is no straightforward relationship between the image size and real size of a small dislocation loop $(<10 \mathrm{~nm})$. However, under well controlled WBDF condition the width at half intensity along the direction of maximum extent of the defect image can be used as an approximation of DL size ${ }^{13}$. Considering two specific DLs, Fig. 11 shows that their apparent size varies significantly from projection to projection. These changes merit further discussion. Under diffraction contrast conditions, changes in the apparent size of DLs can be divided into two categories: Geometric and experimental. Geometric variations refer to size changes caused by viewing of the DL strain field from different angles. In this case, a smooth, sinusoidal variation with tilting angle is expected. Experimental dependence refers to experimental uncertainties that affect the apparent size of a DL, such as localized bending, surface roughness, thickness contours, residual stresses, and change of the distance of a DL to sample surface along the electron beam direction? ${ }^{7}$ These experimental effects are difficult to quantify. The substantial variation of DL size from projection to projection in Fig. 11 suggests that experimental factors play an important role. To reduce the errors incurred from experimental factors, the DL size we report is determined by averaging the apparent size over all 
projections where the DL is visible. The substantial variations in apparent DL size in different projections also advises caution for studies that rely on single WBDF images of a defect microstructure to determine DL size distribution.

(a)
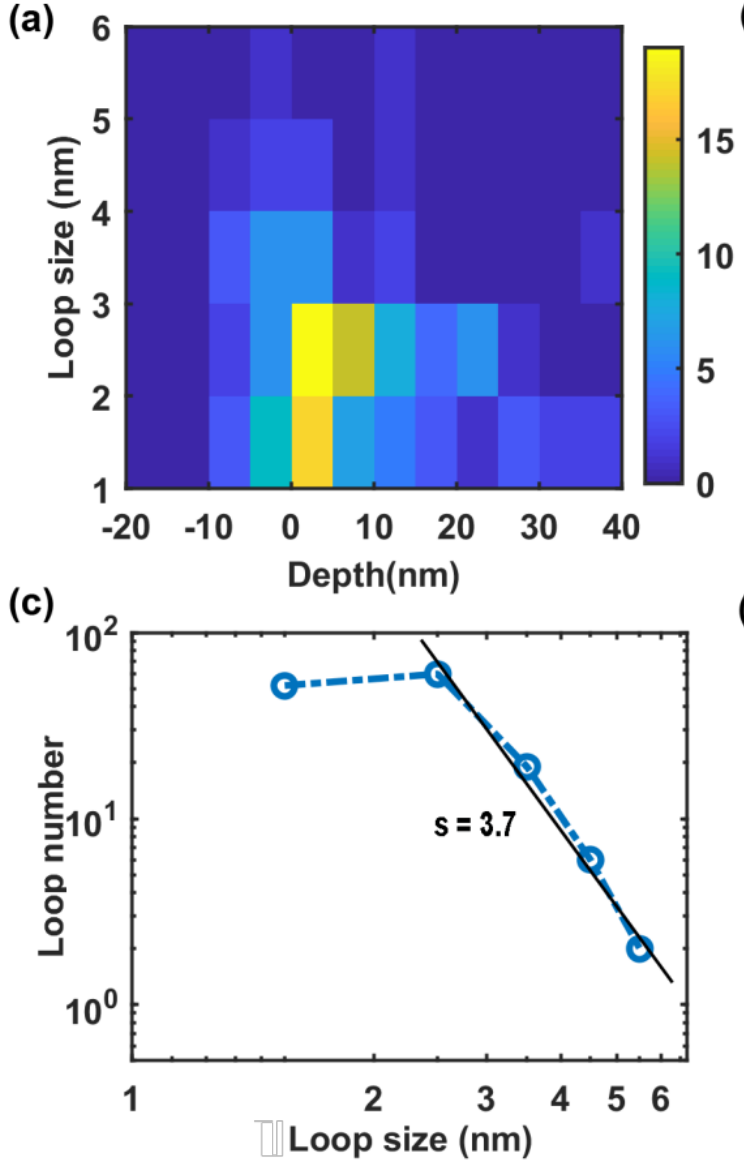

(b)

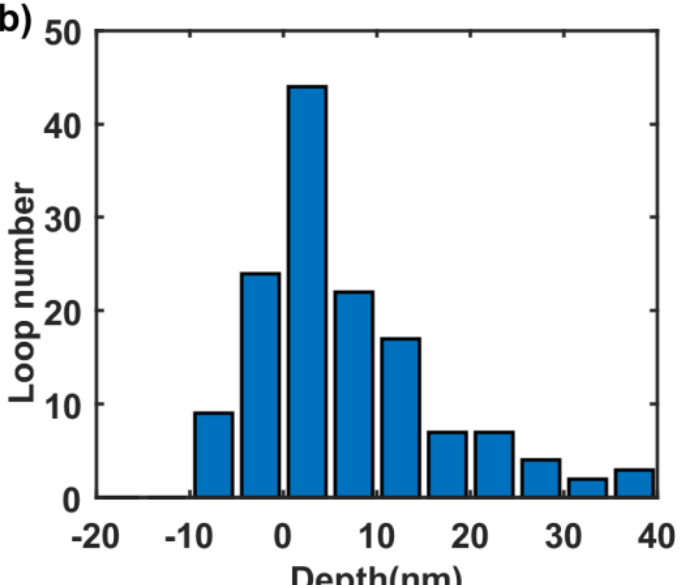

(d)

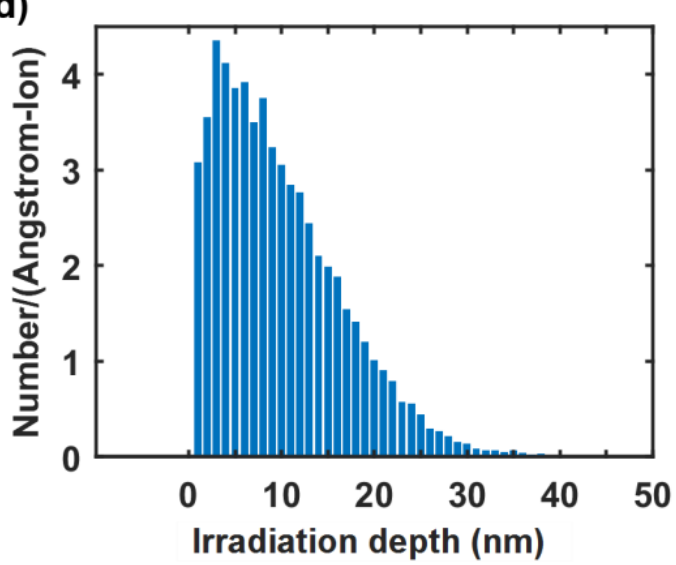

Fig. 12 (a) Number of dislocation loops in the reconstructed volume as a function of depth and loop size. (b) and (c) are histograms obtained by summing the 2D plot of (a) along the dimension of loop size and depth, respectively. The size distribution in (c) is plotted on a log-log scale. (d) Damage profile for $150 \mathrm{keV}$ self-ion implantation of pure W predicted by SRIM.

Now that 3D DL positions and sizes are known, this data can be analysed in more detail. Fig. 12 (a) shows the number of DLs in the reconstructed volume, plotted as a function of loop depth (in $5 \mathrm{~nm}$ increments) and loop size (1 $\mathrm{nm}$ increments). DLs are distributed over a $50 \mathrm{~nm}$ thick region in the depth direction, ranging from $-10 \mathrm{~nm}$ to $40 \mathrm{~nm}$ relative to the rotation centre, where the sample 
surface is at a depth of approximately $-10 \mathrm{~nm}$. DL sizes range from $1 \mathrm{~nm}$ to $6 \mathrm{~nm}$. Interestingly the size distribution of DLs varies as a function of depth in the sample. Furthermore, the depth profile of DL density varies depending on the loop size. This suggests that the depth-dependent variation of defect population is not a simple function of damage dose, information that is lost when only considering 2D WBDF micrographs recorded at a single tilt.

By integrating the 2D plot in Fig. 12 (a) either in the depth or in the loop size directions, histograms of depth dependence of DL population or size distribution can be obtained (12 (b) and (c) respectively). The average number density of dislocation loops is $7.2 \times 10^{21} \mathrm{~m}^{-3}$ (considering a $50 \mathrm{~nm}$ implanted layer thickness). However, the actual number density is different for each layer. The layer from 0 to $5 \mathrm{~nm}$ depth with respect to the rotation center has the highest number density, $2.2 \times 10^{22} \mathrm{~m}^{-3}$. It should be noted that the loop number density reported here is lower than actual number density, as some defects probably will remain invisible in all projections. Fig. 12 (d) shows the damage profile for $150 \mathrm{keV}$ self-ion implantation in $\mathrm{W}$ predicted using the SRIM code. Comparison of Fig. 12 (b) and (d) shows that the anticipated damage profile matches the experimentally observed depth-dependence of DL number density quite well, except within $10 \mathrm{~nm}$ of the free surface. This suggests that the sample surface acts as a strong sink for small defects, an effect that has been predicted by Kinetic Monte Carlo simulations of damage evolution in tungsten ${ }^{15}$. The size distribution of DLs, plotted on a $\log -\log$ scale (Fig. $12(\mathrm{c})$ ), follows a power law $\mathrm{f} \sim \mathrm{A} / \mathrm{d}^{\mathrm{s}}$, where $\mathrm{f}$ is the frequency of occurence, $\mathrm{A}$ is a constant, and $\mathrm{d}$ is the diameter of DL, $\mathrm{s}$ is the exponent. The exponent is 3.7, in good agreement with predictions from MD simulations ${ }^{46}$. For loops smaller than $2 \mathrm{~nm}$ a deviation from this power law is observed, in agreement with previous observations from 2D micrographs ${ }^{14}$. This may be due to a lack of sensitivity of WBDF TEM measurements to these very small defects, which has been previously pointed out ${ }^{34}$. 


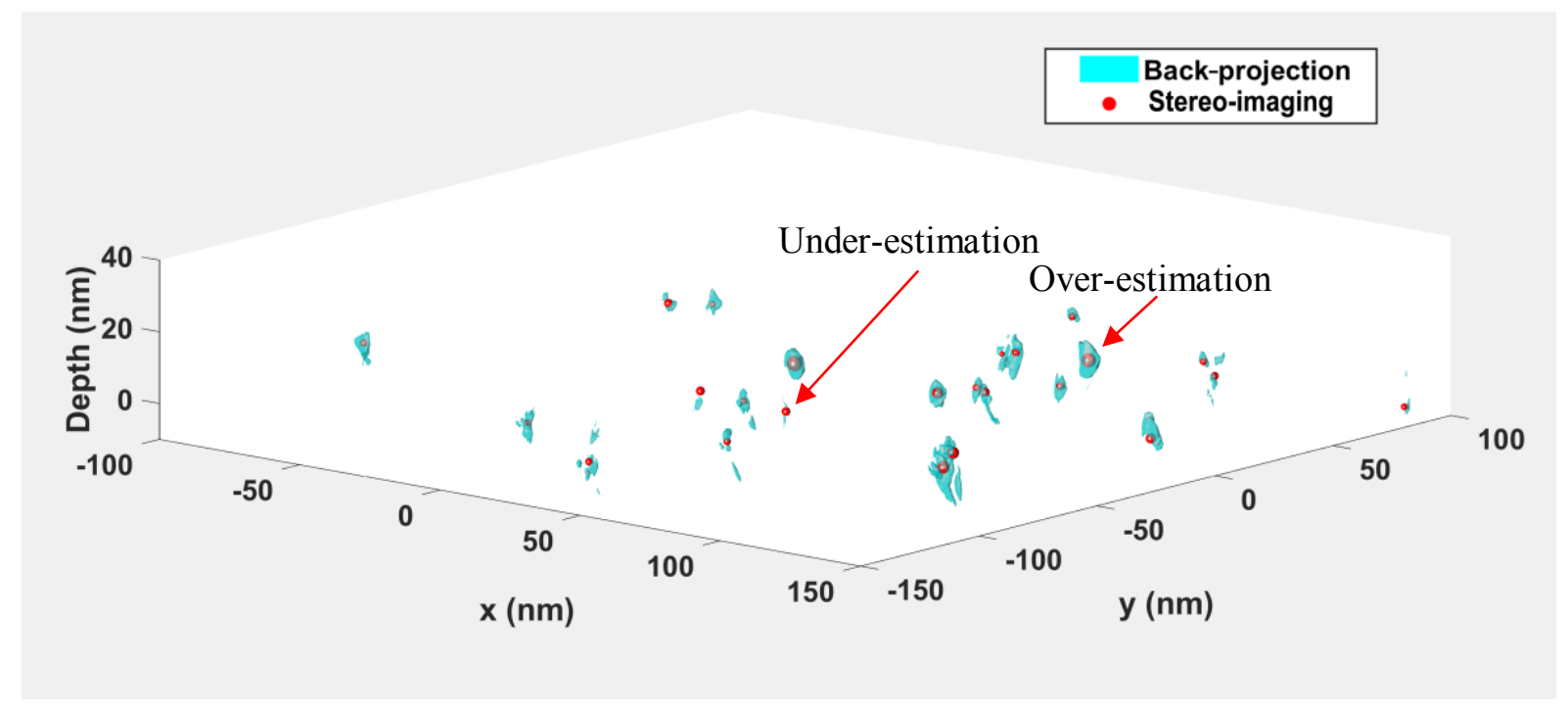

Fig. 13 Superposition of the 3D reconstructions by automated stereo-imaging approach and weighted back-projection.

Fig. 13 shows a comparison of the spatial distribution of DLs, recovered using the automated stereoimaging approach, with the damage microstructrue recovered by weighted back-projection (see supplementary movie 3 for 3D animation). Qualitatively the 3D spatial distribution of DLs agrees well. However, misalignment (random shift of individual loop in each projection) and missing wedge artefacts can lead to an over-estimation of loop size from backprojection. For other loops, missing projections, which cannot be accounted for on a loop-by-loop basis in the weighted back-projection scheme, cause the underestimation of the size. Fig. 13 also shows that weighted back-projection yields somewhat surprising, likely spurrious, loop morphologies. The stereo-imaging approach, where loop size is determined based on the apparent size of dislocation loops in 2D projections, avoides this effect. This benefit was also noted in a recent study where use of a stereo-imaging approach reduced the reconstruction errors for line dislocations ${ }^{47}$. 


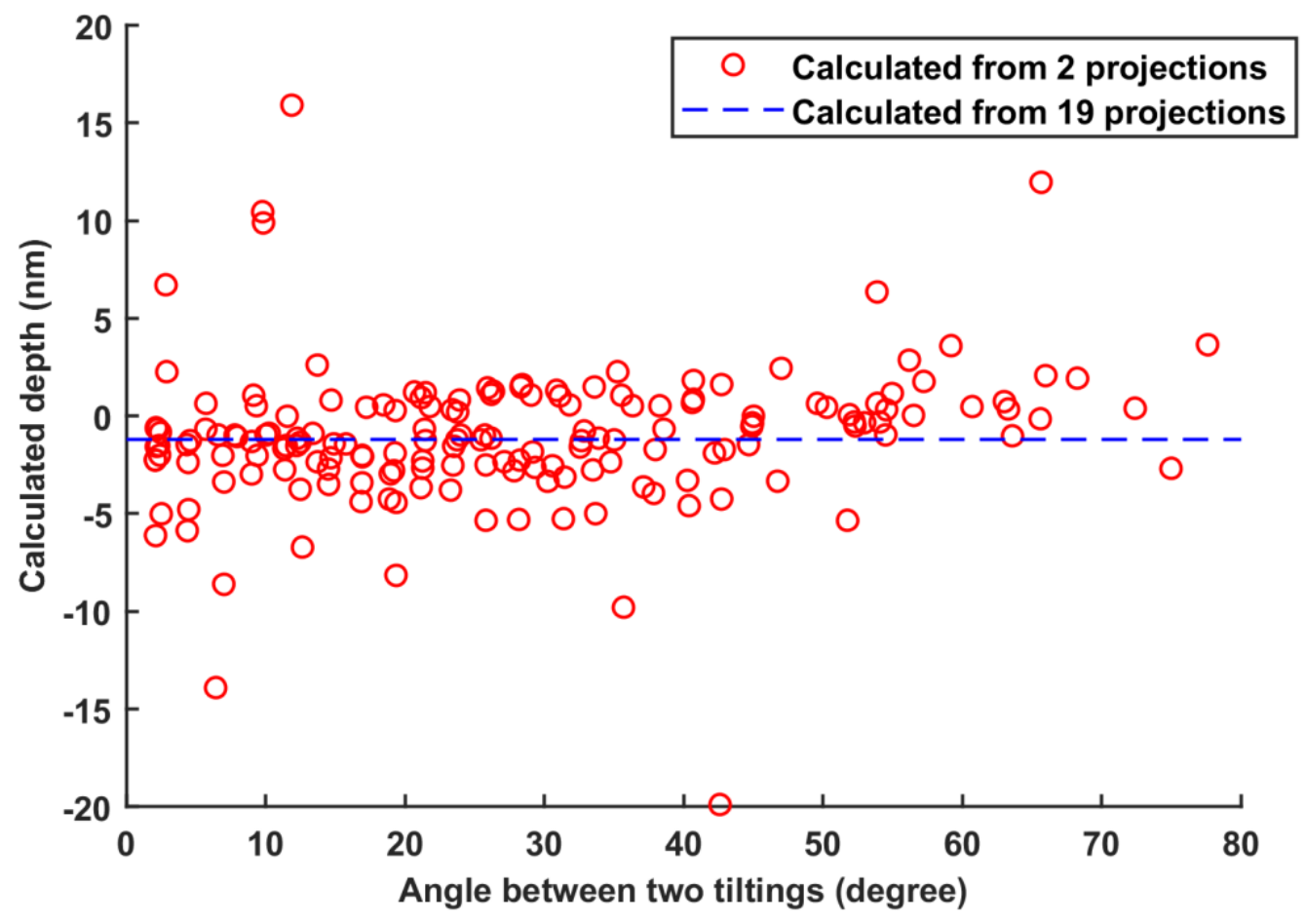

Fig. 14 The estimated depth of a specific DL versus the angle between the two projections used to estimate the depth of the DL. Superimposed is a dashed line indicating the depth determined by fitting of all 19 projections.

The angular range over which projections are collected is also expected to play an important role, with a small tilting range reducing the accuracy of depth determination. Fig. 14 shows a plot of the depth of a DL, estimated from two projections, plotted as a function of tilting angle change between the two projections. Several outliers are present at angles below 15 degrees. A larger angles, above 50 degrees, an increase in scatter is also evident. This is mainly due to localized bending, which makes it difficult to maintain exactly the same diffraction condition when two projections are recorded at very different tilting angles. However, overall, the estimated depth shows surprisingly little dependence on the angle between the two projections. Thus, since the high angle tilting required for conventional reconstruction scheme is not needed for the stereo-imaging approach, an angular tilt range of 30 to 40 degrees provides a good compromise. 


\subsection{Applicability and limitations of the automated stereo-imaging approach}

The automated stereo-imaging approach is applicable to cases where the features to be reconstructed are compact objects in space. Here we assumed that the small DLs formed by self-ion irradition have spherical morphology. This prior knowledge reduces the number of projections required. However, in principle objects with more complex morphology could be consider, so long as their morphology can be readily parameterised, e.g. elipsoids. This would allow extension of the approach to more complex geometries, such as larger dislocation loops. The robutness and faithfullness of the reconstruction depends on the projected number density of features and level of imperfections of projections. Errors in the mechanical tilting angle control will also contribute the reconstrution error ${ }^{48}$, advising use of a calibrated tilting holder.

Here, the projection images of the dislocation loops for each tilting angle were recorded using a single WBDF diffraction condition. It is important to note that, in WBDF imaging of small loops, the size and visibily of loops are very sensitive to $\mathrm{Sg}_{\mathrm{g}}{ }^{7}$ (see Figs. S2 and 11). This effect can be reduced by using convergent WBDF ${ }^{49}$ or precession of the electron beam ${ }^{50-52}$. In both cases the images with slightly varying $\mathrm{s}_{\mathrm{g}}$ are recorded and superimposed, which can significantly reduce size and visibility variation.

The minimum number of projections required for reconstruction is strongly dependent on the projected number density and experimental errors. Based on the synthetic data, when the projection number density is in the range of $5 \times 10^{15} \mathrm{~m}^{-2}, 19$ projections are required for reconstruction with acceptable errors $(<5 \%)$. Fewer projections are required when the density is lower or the experimental errors are reduced (e.g. using a high quality sample, or employing convergent WBDF or precession of the electron beam). More projections will be needed if a greater number density is of interest. 


\section{Conclusions}

We have developed an automated registration and stereo-imaging approach for determining the 3D distribution of small dislocation loops from weak-beam dark field TEM micrographs recorded at a number of different tilt angles. As a demonstration, this approach is applied to the study of the 3D damage microstructure formed in low-dose self-ion implanted tungsten. Using a forward prediction approach, each specific DL is identified in all the projections. A system of linear equations is then setup linking the 3D position of each DL to its 2D position in each projection. Least-squares optimisation is used to find a solution for the 3D loop position. Using this $3 \mathrm{D}$ information the spatial correlation between defects, as well as size and depth-dependence of the dislocation loop population, can be examined. Our analysis shows that the automated stereo-imaging approach is more tolerant to contrast variations than back-projection approaches and requires relatively fewer projections that span a more modest tilting angle range.

\section{Acknowledgements}

We thank Daniel Mason for help with image processing, Marquis Kirk, Pete Baldo and Edward Ryan for their help with the irradiations, and Meimie Li for helpful discussion. We acknowledge funding from the European Research Council (ERC) under the European Union's Horizon 2020 research and innovation programme (grant agreement No 714697), and funding from the Oxford University John Fell Fund (grant 161/054). The experiments of in situ ion irradiations at liquid helium temperature were carried out at Argonne National Laboratory (ANL), using the IVEM-Tandem Facility. These experiments in ANL were supported by a U.S. Department of Energy Facility funded by the DOE Office of Nuclear Energy, operated under Contract No. DEAC02-06CH11357 by U. Chicago Argonne, LLC. The Matlab reconstruction code written for this project is available online DOI: 10.5281/zenodo.1402439. 


\section{Appendix}

Given a unit vector $\mathbf{r}=\left(\mathrm{r}_{1}, \mathrm{r}_{2}, \mathrm{r}_{3}\right)$, where $\mathrm{r}_{1}^{2}+\mathrm{r}_{2}^{2}+\mathrm{r}_{3}^{2}=1$, the transformation matrix for a rotation by an angle of $\theta$ about $\mathbf{r}$, following a right-handed convention, is given by

$$
R=\left[\begin{array}{ccc}
r_{1}^{2}(1-\cos \theta)+\cos \theta & r_{1} r_{2}(1-\cos \theta)-r_{3} \sin \theta & r_{1} r_{3}(1-\cos \theta)+r_{2} \sin \theta \\
r_{2} r_{1}(1-\cos \theta)+r_{3} \sin \theta & r_{2}^{2}(1-\cos \theta)+\cos \theta & r_{2} r_{3}(1-\cos \theta)-r_{1} \sin \theta \\
r_{3} r_{1}(1-\cos \theta)-r_{2} \sin \theta & r_{3} r_{2}(1-\cos \theta)+r_{1} \sin \theta & r_{3}^{2}(1-\cos \theta)+\cos \theta
\end{array}\right]
$$

\section{References}

1. Was, G. S. Irradiation Hardening and Deformation. in Fundamentals of Radiation Materials Science: Metals and Alloys 581-642 (Springer Berlin Heidelberg, 2007). doi:10.1007/978-3540-49472-0_12

2. Armstrong, D. E. J., Yi, X., Marquis, E. A. \& Roberts, S. G. Hardening of self ion implanted tungsten and tungsten 5-wt\% rhenium. J. Nucl. Mater. 432, 428-436 (2013).

3. Yao, Z., Hernández-Mayoral, M., Jenkins, M. L. \& Kirk, M. A. Heavy-ion irradiations of Fe and Fe-Cr model alloys Part 1: Damage evolution in thin-foils at lower doses. Philos. Mag. 88, $2851-2880$ (2008).

4. Li, M., Kirk, M. A., Baldo, P. M., Xu, D. \& Wirth, B. D. Study of defect evolution by TEM with in situ ion irradiation and coordinated modeling. Philos. Mag. 92, 2048-2078 (2012).

5. Yi, X. et al. In situ study of self-ion irradiation damage in $\mathrm{W}$ and $\mathrm{W}-5 \mathrm{Re}$ at $500{ }^{\circ} \mathrm{C}$. Philos. Mag. 93, 1715-1738 (2013).

6. Jenkins, M. L. Characterisation of radiation-damage microstructures by TEM. J. Nucl. Mater. 216, 124-156 (1994).

7. Jenkins, M. L. \& Kirk, M. A. Characterization of radiation-damage by transmission electron microscopy. (Institute of Physics Pub, 2001).

8. Yao, Z. \& Ken Zhang, H. In-situ TEM Observation of Nanoscale Stacking Fault Tetrahedra in A Ni Based Superalloy Inconel X-750. in Microscopy: advances in scientific research and 
education (A. Méndez-Vilas, Ed.) 842-849 (2014).

9. $\mathrm{Yu}$, H. et al. Accumulation of dislocation loops in the $\alpha$ phase of $\mathrm{Zr}$ Excel alloy under heavy ion irradiation. J. Nucl. Mater. 491, 232-241 (2017).

10. Kirk, M., Yi, X. \& Jenkins, M. Characterization of irradiation defect structures and densities by transmission electron microscopy. J. Mater. Res. 30, 1195-1201 (2015).

11. Zhang, H. K., Yao, Z., Judge, C. \& Griffiths, M. Microstructural evolution of CANDU spacer material Inconel X-750 under in situ ion irradiation. J. Nucl. Mater. 443, 49-58 (2013).

12. Yi, X., Jenkins, M. L., Hattar, K., Edmondson, P. D. \& Roberts, S. G. Characterisation of radiation damage in $\mathrm{W}$ and $\mathrm{W}$-based alloys from $2 \mathrm{MeV}$ self-ion near-bulk implantations. Acta Mater. 92, 163-177 (2015).

13. Kirk, M., Yi, X. \& Jenkins, M. Characterization of irradiation defect structures and densities by transmission electron microscopy. J. Mater. Res. 30, 1195-1201 (2015).

14. Yi, X. et al. Direct observation of size scaling and elastic interaction between nano-scale defects in collision cascades. Epl 110, 36001 (2015).

15. Mason, D. R., Yi, X., Kirk, M. A. \& Dudarev, S. L. Elastic trapping of dislocation loops in cascades in ion-irradiated tungsten foils. J. Phys. Condens. Matter 26, (2014).

16. Mason, D. R., Sand, A. E., Yi, X. \& Dudarev, S. L. Direct observation of the spatial distribution of primary cascade damage in tungsten. Acta Mater. 144, 905-917 (2018).

17. Rühle, M., F. Häussermann \& Rapp, M. Transmission Electron Microscopy of Point-Defect Clusters in Neutron-Irradiated Metals. Phys. Status Solidi B 39, 609 (1970).

18. Mcintyre, K. G. Interstitial dislocation loops in neutron irradiated copper. Philos. Mag. 15, 205-208 (1967).

19. B. Hudson. The application of stereo-techniques to electron micrographs. 98, 396-401 (1973).

20. Maher, D. M., Loretto, M. H. \& Bartlett, A. F. Neutron irradiation damage in molybdenum. Philos. Mag. 24, 181-194 (1971). 
21. Hasanzadeh, S. et al. Three-dimensional scanning transmission electronmicroscopy of dislocation loops in tungsten. Micron Accepted, (2018).

22. Baumeister, W. Electron tomography: Towards visualizing the molecular organization of the cytoplasm. Curr. Opin. Struct. Biol. 12, 679-684 (2002).

23. Gilbert, P. Iterative Methods for the Three-dimensional Reconstruction of an Object from Projections. J. theor. Biol. 36, 105-117 (1972).

24. Batenburg, K. J. et al. 3D imaging of nanomaterials by discrete tomography. Ultramicroscopy 109, 730-740 (2009).

25. Midgley, P. A. \& Dunin-Borkowski, R. E. Electron tomography and holography in materials science. Nat. Mater. 8, 271-280 (2009).

26. Chen, D. et al. The properties of SIRT, TVM, and DART for 3D imaging of tubular domains in nanocomposite thin-films and sections. Ultramicroscopy 147, 137-148 (2014).

27. Goris, B., Van den Broek, W., Batenburg, K. J., Heidari Mezerji, H. \& Bals, S. Electron tomography based on a total variation minimization reconstruction technique. Ultramicroscopy 113, $120-130(2012)$.

28. Leary, R., Saghi, Z., Midgley, P. A. \& Holland, D. J. Compressed sensing electron tomography. Ultramicroscopy 131, 70-91 (2013).

29. Saghi, Z. et al. Three-dimensional morphology of iron oxide nanoparticles with reactive concave surfaces. A compressed sensing-electron tomography (CS-ET) approach. Nano Lett. 11, 4666-4673 (2011).

30. Kacher, J. \& Robertson, I. M. In situ TEM characterisation of dislocation interactions in $\alpha-$ titanium. Philos. Mag. 96, 1437-1447 (2016).

31. Kacher, J. P., Liu, G. S. \& Robertson, I. M. Visualization of grain boundary/dislocation interactions using tomographic reconstructions. Scr. Mater. 64, 677-680 (2011).

32. Barnard, J. S., Sharp, J., Tong, J. R. \& Midgley, P. A. High-resolution three-dimensional 
imaging of dislocations. Science (80-. ). 313, 319 (2006).

33. Z.Q., F. et al. Electron tomography of dislocations in an $\mathrm{Al}-\mathrm{Cu}-\mathrm{Mg}$ alloy. IOP Conf. Ser. Mater. Sci. Eng. 219, 12018 (2017).

34. Zhou, Z., Jenkins, M. L., Dudarev, S. L., Sutton, A. P. \& Kirk, M. A. Simulations of weakbeam diffraction contrast images of dislocation loops by the many-beam Howie-Basinski equations. Philosophical Magazine 86, (2006).

35. Ziegler, J. F. INTERACTIONS OF IONS WITH MATTER. http://www.srim.org/

36. Williams, G. Overdetermined Systems of Linear Equations. Am. Math. Mon. 97, 511-513 (1990).

37. Pan, B., Qian, K., Xie, H. \& Asundi, A. Two-dimensional digital image correlation for inplane displacement and strain measurement: a review. Meas. Sci. Technol. 20, 62001 (2009).

38. Lowe, D. G. Object recognition from local scale-invariant features. in Proceedings of the Seventh IEEE International Conference on Computer Vision 2, 1150-1157 vol.2 (1999).

39. Lowe, D. G. Distinctive Image Features from Scale-Invariant Keypoints. Int. J. Comput. Vis. 60, 91-110 (2004).

40. Nixon, M. S. \& Aguado, A. S. Feature extraction \& image processing for computer vision. (Academic Press, 2012).

41. Tony Lindeberg. Scale-Space Theory in Computer Vision. (Springer US, 1994). doi:10.1007/978-1-4757-6465-9

42. Ridler, T. W. \& Calvard, S. Picture Thresholding Using an Iterative Selection Method. IEEE Trans. Syst. Man. Cybern. 8, 630-632 (1978).

43. Gonzalez, R. C. \& Woods, R. E. Digital Image Processing (3rd Edition). (Prentice-Hall, Inc., 2006).

44. Radermacher, M. Weighted Back-projection Methods. in Electron Tomography: Methods for Three-Dimensional Visualization of Structures in the Cell (ed. Frank, J.) 245-273 (Springer 
New York, 2006). doi:10.1007/978-0-387-69008-7_9

45. Sage, D. et al. DeconvolutionLab2: An open-source software for deconvolution microscopy. Methods 115, 28-41 (2017).

46. Sand, A. E., Dudarev, S. L. \& Nordlund, K. High-energy collision cascades in tungsten: Dislocation loops structure and clustering scaling laws. Epl 103, (2013).

47. Oveisi, E. et al. Stereo-vision three-dimensional reconstruction of curvilinear structures imaged with a TEM. Ultramicroscopy 184, 116-124 (2018).

48. Hudson, B. \& Makin, M. J. The optimum tilt angle for electron stereo- microscopy The optimum tilt angle for electron. J. Phys. E Sci. Instrum. 3, 311 (1970).

49. Schäublin, R., Meng, X. \& Stobbs, W. M. Weak beam under convergent beam illumination. Ultramicroscopy 83, 145-157 (2000).

50. Prokhodtseva, A., Décamps, B., Ramar, A. \& Schäublin, R. Impact of He and Cr on defect accumulation in ion-irradiated ultrahigh-purity Fe ( Cr ) alloys. Acta Mater. 61, 6958-6971 (2013).

51. Rebled, J. M., Yedra, L., Estrade, S., Portillo, J. \& F. Peiro' a, N. A new approach for 3D reconstruction from bright field TEM imaging: Beam precession assisted electron tomography. Ultramicroscopy 111, 1504-1511 (2011).

52. Barnard, J. S. et al. Dislocation electron tomography and precession electron diffraction minimising the effects of dynamical interactions in real and reciprocal space. Philos. Mag. 6435, (2010). 


\section{Supplementary material for:}

3D reconstruction of the spatial distribution of dislocation loops using an automated stereo-imaging approach Hongbing $\mathrm{Yu}{ }^{1}$, Xiaoou $\mathrm{Yi}^{2}$, Felix Hofmann ${ }^{1}$

(1) Department of Engineering Science, University of Oxford, Parks Road, Oxford, OX1 3PJ, UK

(2) School of Materials Science and Engineering, University of Science and Technology Beijing, Beijing, 100083, China

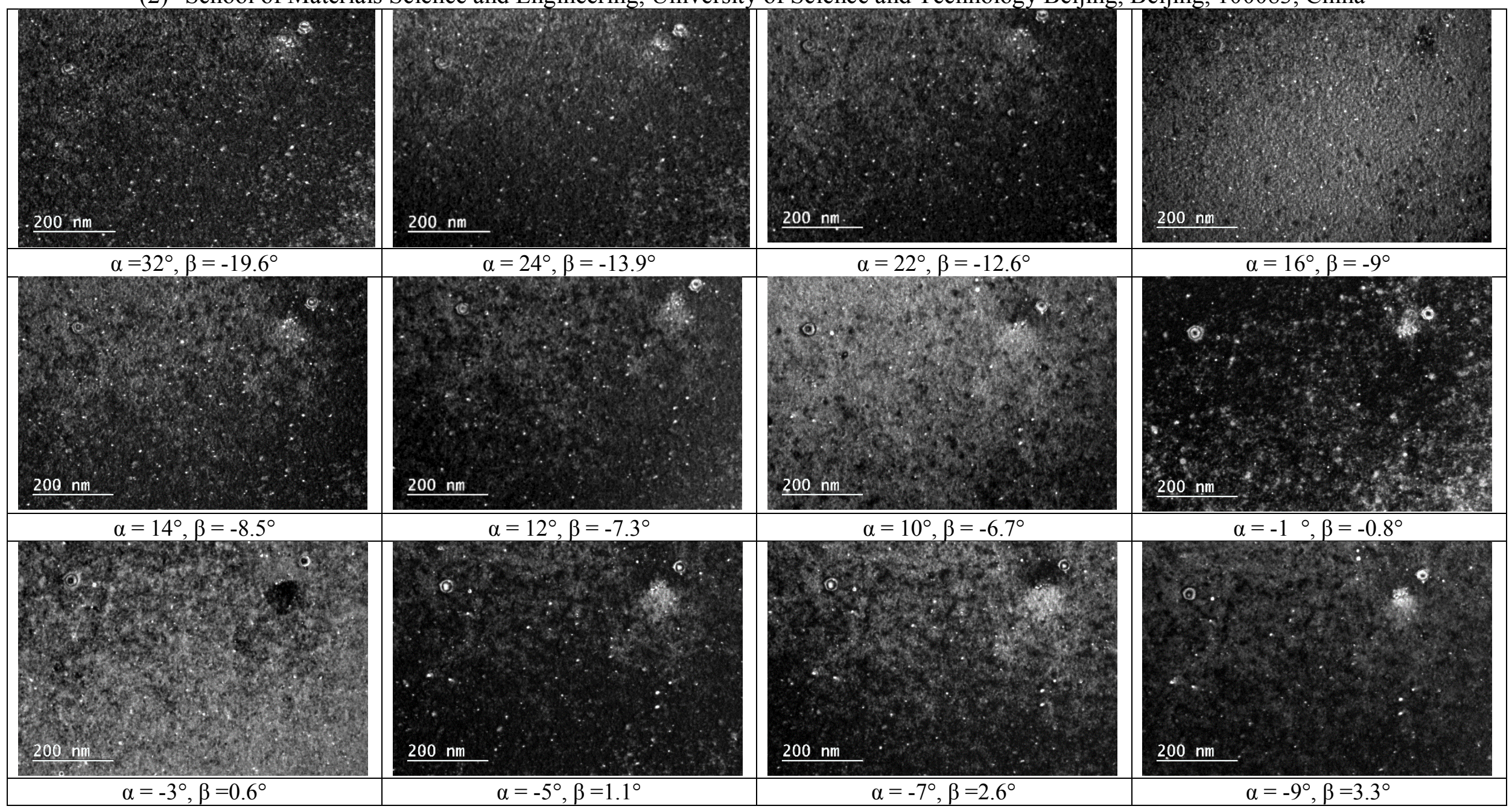




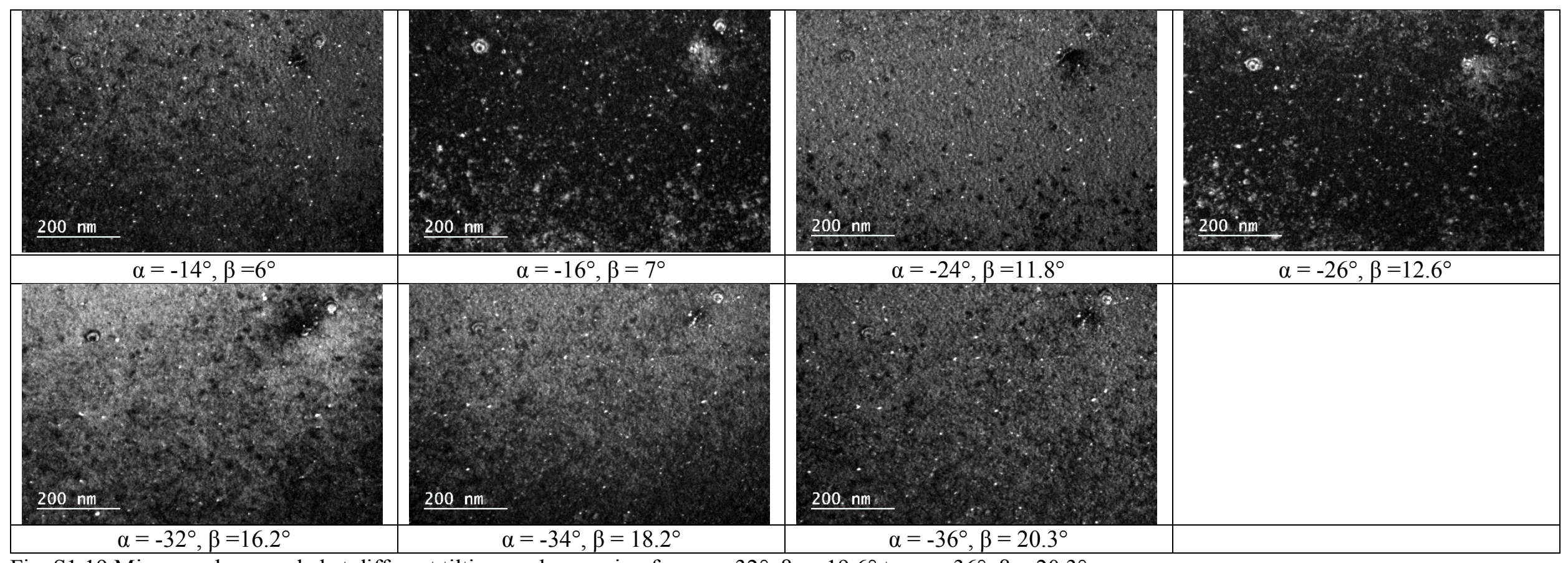

Fig. S1 19 Micrographs recorded at different tilting angles ranging from $\alpha=32^{\circ}, \beta=-19.6^{\circ}$ to $\alpha=-36^{\circ}, \beta=20.3^{\circ}$. 


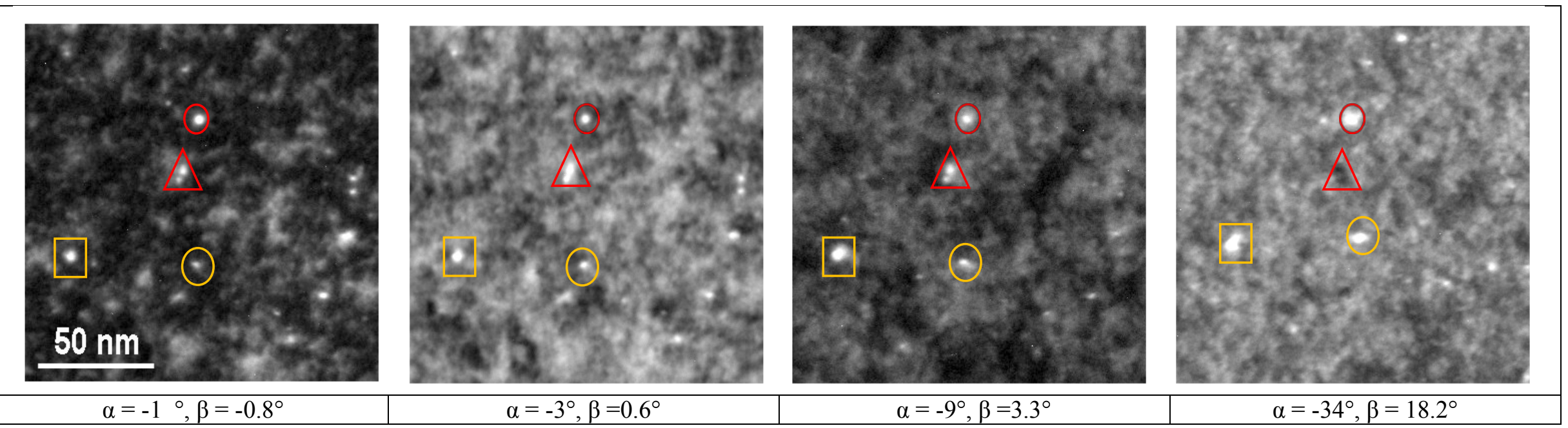

Fig. S2 A small area cropped out from 4 of the 19 recorded micrographs, demonstrating variation of the morphology, size, intensity and visibility of specific dislocation loops with tilting angle. 


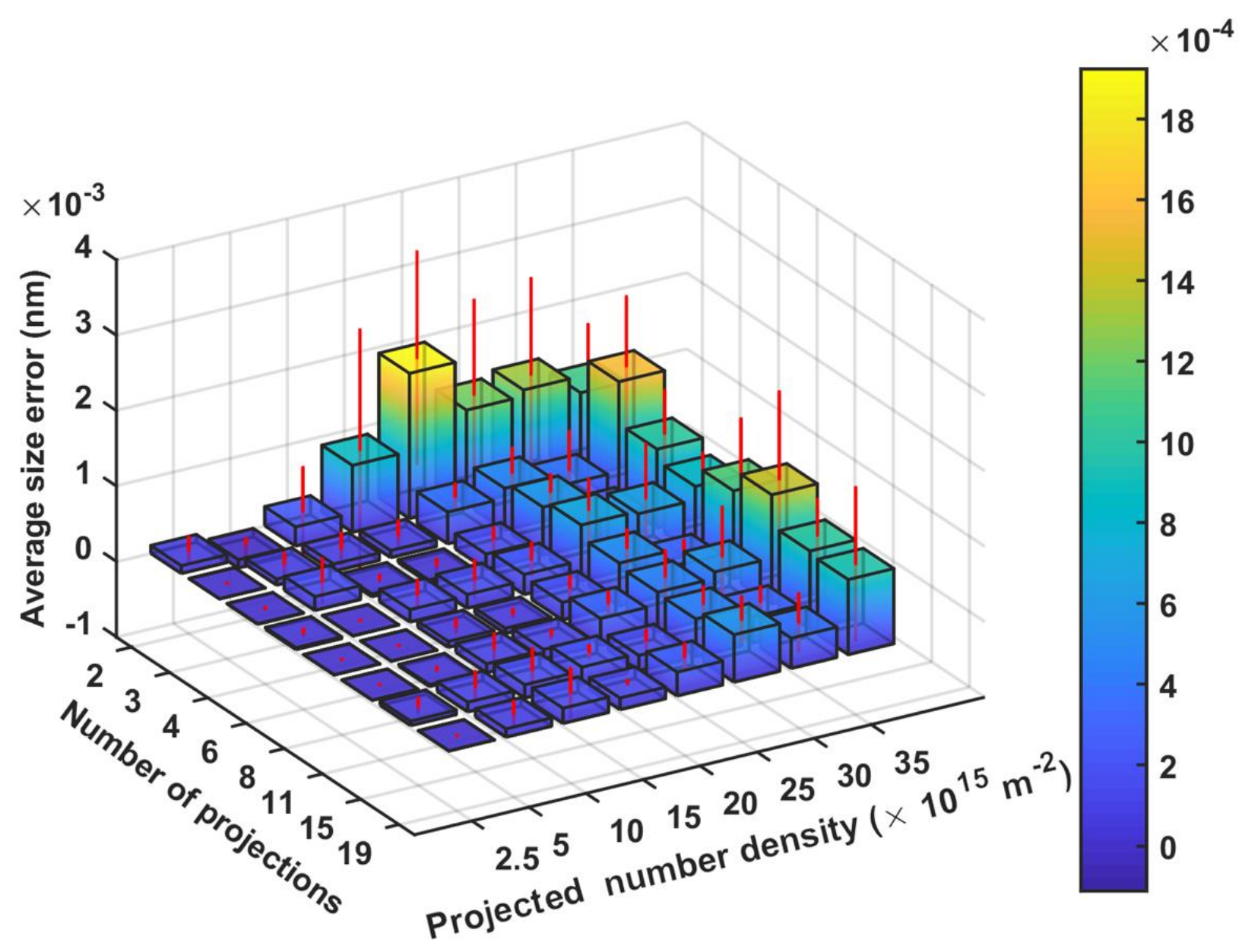

Fig. S3 The average size error as a function of number of projections and projected number densities of loops for the case of perfect projections. 


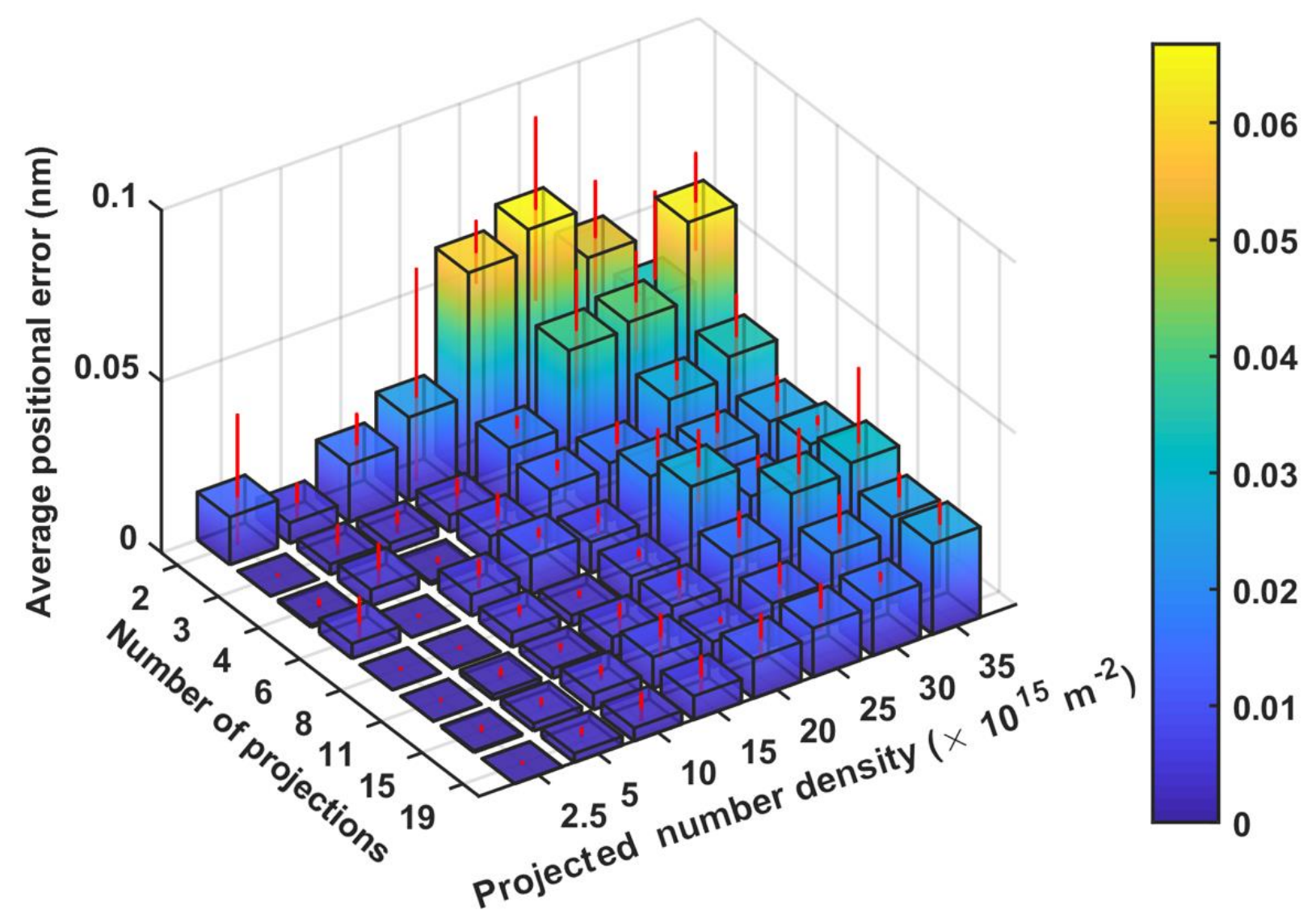

Fig. S4 The average position error as a function of number of projections and projected number densities of loops for the case of perfect projections. 


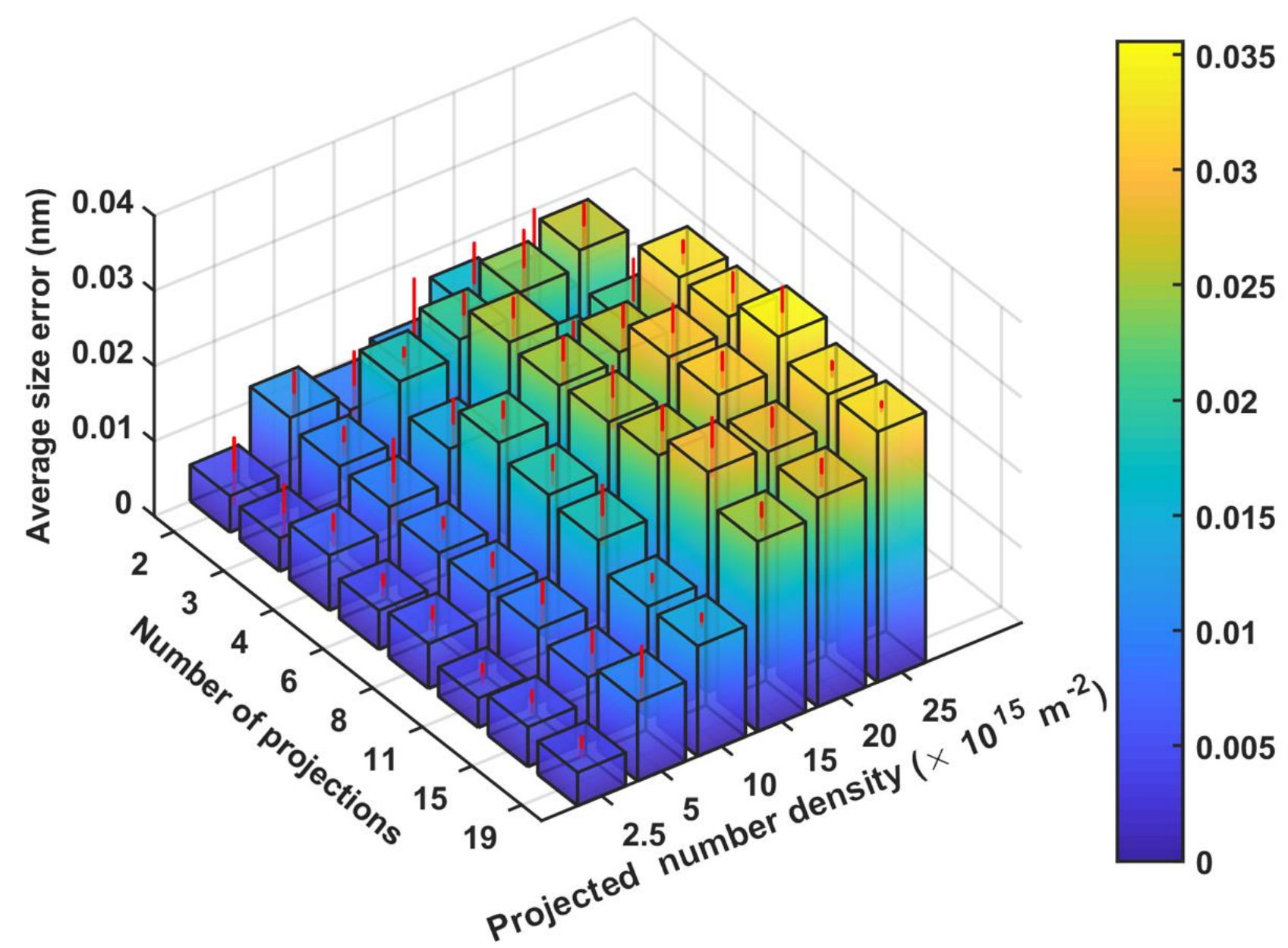

Fig. S5 The average size error as a function of number of projections and projected number densities of loops for the case for the case where random position shifts were added to the projections of loops. 


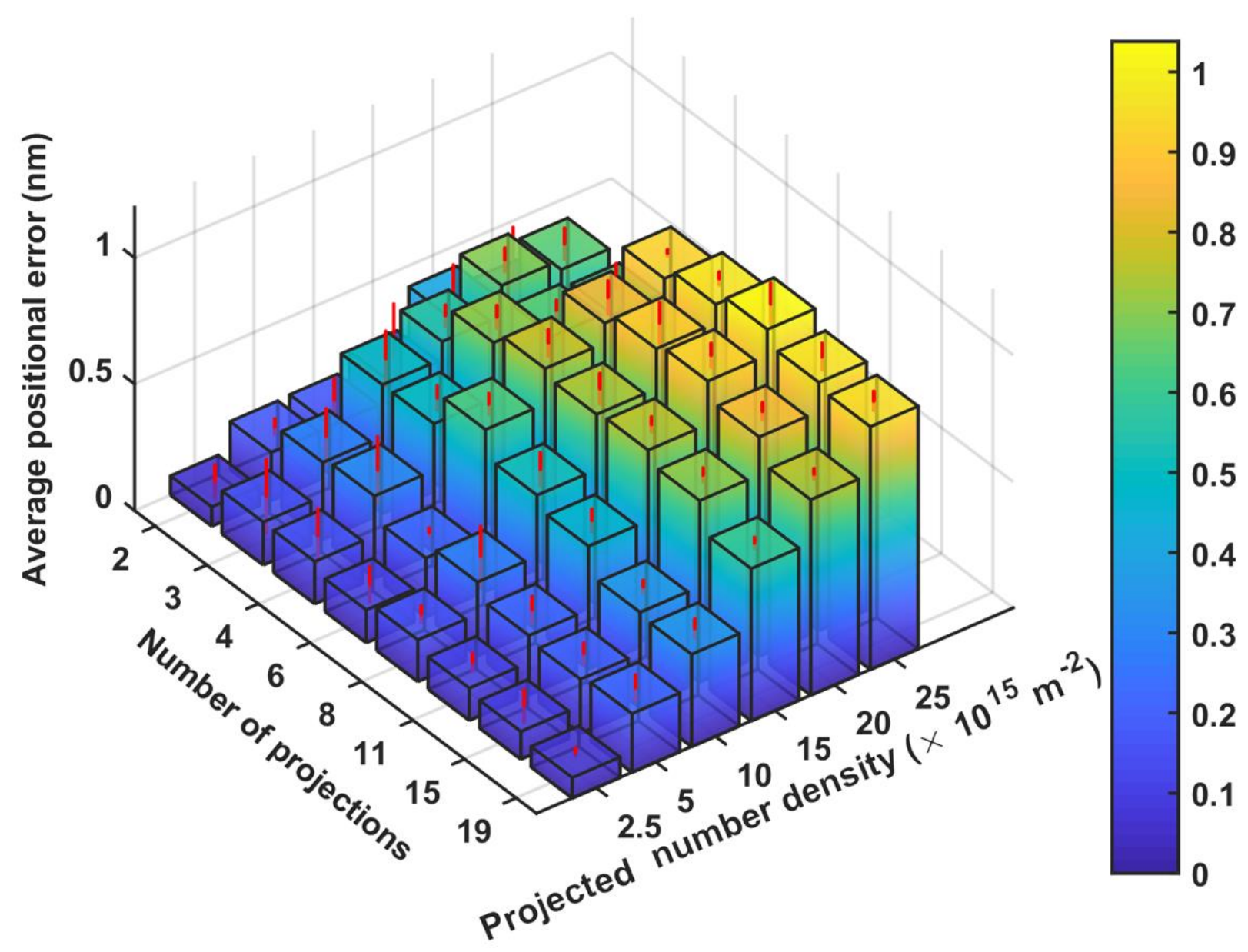

Fig. S6 The average position error as a function of number of projections and projected number densities of loops for the case for the case where random position shifts were added to the projections of loops. 